\title{
Multistage Stochastic Programming Approach for Offshore Oilfield Infrastructure Planning under Production Sharing Agreements and Endogenous Uncertainties
}

\author{
Vijay Gupta*, Ignacio E. Grossmann†, \\ Department of Chemical Engineering, Carnegie Mellon University \\ Pittsburgh, PA 15213
}

\begin{abstract}
The paper presents a new optimization model and solution approach for the investment and operations planning of offshore oil and gas field infrastructure. As compared to previous models where fiscal rules and uncertainty in the field parameters are considered separately, the proposed model is the first one in the literature that includes both of these complexities in an efficient manner. In particular, a tighter formulation for the production sharing agreements is used based on our recent work, and correlation among the endogenous uncertain parameters (field size, oil deliverability, water-oil ratio and gas-oil ratio) is considered to reduce the total number of scenarios in the resulting multistage stochastic formulation. To solve large instances, a Lagangean decomposition approach allowing parallel solution of the scenario subproblems is implemented in the GAMS grid computing environment. Computational results on a variety of oilfield development planning examples are presented to illustrate the efficiency of the model and the proposed solution approach.
\end{abstract}

Keywords: multistage stochastic programming; endogenous uncertainties; non-anticipativity constraints; Lagrangean decomposition; oil \& gas exploration, production sharing agreements

*E-mail: vijaygup@andrew.cmu.edu

†To whom all correspondence should be addressed. E-mail: grossmann@cmu.edu 


\section{Introduction}

The life cycle of a typical offshore oilfield project consists of the following five steps:

(1) Exploration: This activity involves geological and seismic surveys followed by exploration wells to determine the presence of oil or gas.

(2) Appraisal: It involves drilling of delineation wells to establish the size and quality of the potential field. Preliminary development planning and feasibility studies are also performed.

(3) Development: Following a positive appraisal phase, this phase aims at selecting the most appropriate development plan among many alternatives. This step involves capitalintensive investment and operating decisions that include facility installations, drilling, sub-sea structures, etc.

(4) Production: After the facilities are built and wells are drilled, production starts where gas or water is usually injected in the field at a later time to enhance productivity.

(5) Abandonment: This is the last phase of an oilfield development project and involves the decommissioning of facility installations and subsea structures associated with the field.

Given that most of the critical investments are usually associated with the development planning phase of the project, this paper focus is on the key strategic/tactical decisions during this phase of the project. The major decisions involved in the oilfield development planning phase are the following:

(i) Selecting platforms to install and their sizes

(ii) Deciding which fields to develop and the order to develop them

(iii) Deciding which wells and how many are to be drilled in the fields and in what sequence

(iv) Deciding which fields are to be connected to what facility

(v) Determining how much oil and gas to produce from each field

Therefore, there are a very large number of alternatives that are available to develop a particular field or group of fields. However, these decisions should account for the physical and practical considerations, such as the following: a field can only be developed if a corresponding facility is present; nonlinear profiles of the reservoir that are obtained from reservoir simulators (e.g. ECLIPSE, 2008) to predict the actual flowrates of oil, water and gas from each field; limitation on the number of wells that can be drilled each year due to availability of the drilling rigs; and long-term planning horizon that is the characteristic of these projects. Therefore, 
optimal investment and operating decisions are essential for this problem to ensure the highest return on the investments over the time horizon considered. By including all the considerations described here in an optimization model, this leads to a large-scale multiperiod mixed-integer nonlinear programming (MINLP) problem that is difficult to solve to global optimality. The extension of this model to the cases that explicitly consider the fiscal rules with the host government and the uncertainties can further lead to a very complex problem to model and solve.

In terms of the deterministic approaches, the oilfield development planning has been modeled as linear programming (LP) (Lee and Aranofsky, 1958; and Aronofsky and Williams, 1962) or mixed-integer linear programming (MILP) (Frair, 1973) models under certain assumptions to make them computationally tractable. Simultaneous optimization of the investment and operating decisions has been addressed in Bohannon (1970), Sullivan (1982) and Haugland et al. (1988) using MILP formulations with different levels of details. Behrenbruch (1993) emphasized the need to consider a correct geological model and to incorporate flexibility into the decision process for an oilfield development project. Iyer et al. (1998) proposed a multiperiod MILP model for optimal planning and scheduling of offshore oilfield infrastructure investment and operations. The model considers the facility allocation, production planning, and scheduling within a single model and incorporates the reservoir performance, surface pressure constraints, and oil rig resource constraints. Van den Heever and Grossmann (2000) extended the work of Iyer et al. (1998) and proposed a multiperiod generalized disjunctive programming model for oil field infrastructure planning for which they developed a bilevel decomposition method. As opposed to Iyer and Grossmann (1998), they explicitly incorporated a nonlinear reservoir model into the formulation but did not consider the drill-rig limitations. Barnes et al. (2002) optimized the production capacity of a platform and the drilling decisions for wells associated with this platform. The authors addressed the problem by solving a sequence of MILPs. Ortiz-Gomez et al. (2002) presented three mixed-integer multiperiod optimization models of varying complexity for the oil production planning. Carvalho and Pinto (2006a) considered an MILP formulation for oilfield planning based on the model developed by Tsarbopoulou (2000), and proposed a bilevel decomposition algorithm for solving large-scale problems where the master problem determines the assignment of platforms to wells and a planning subproblem calculates the timing for the fixed assignments. The work was further extended by Carvalho and Pinto (2006b) to consider multiple reservoirs within the model. 
Recently, Gupta and Grossmann (2012a) proposed a general multiperiod MINLP formulation for offshore oilfield development planning that simultaneously optimizes facility installation, well drilling, and production decisions considering oil, water and gas flows profiles. To solve the resulting non-convex MINLP problem, they reformulated it as an MILP using two theoretical properties and piecewise-linear approximations.

The major limitation with the above approaches is that they do not consider the fiscal rules explicitly in the optimization model that are associated to these fields, and mostly rely on the simple net present value (NPV) as an objective function. Therefore, the models with these objectives may yield the solutions that are very optimistic, which can in fact be suboptimal after considering the impact of fiscal terms. Van den Heever et al. (2000) and Van den Heever and Grossmann (2001) considered optimizing the complex economic objectives including royalties, tariffs, and taxes for the multiple gas field site where the schedule for the drilling of wells was predetermined as a function of the timing of the installation of the well platform. Based on a continuous time formulation for gas field development with complex economics of similar nature as Van den Heever and Grossmann (2001), Lin and Floudas (2003) proposed an MINLP model and solved it with a two-stage algorithm. Approaches based on simulation (Blake and Roberts, 2006) and meta-modeling (Kaiser and Pulsipher, 2004) have also been considered for the analysis of the different fiscal terms. Gupta and Grossmann (2012b) presented a generalized mathematical framework and tighter formulations to incorporate a variety of fiscal contracts efficiently in the development planning.

In the literature work described so far, one of the major assumptions is that there is no uncertainty in the model parameters, which in practice is generally not true. Although limited, there has been some work that accounts for uncertainty in the problem of optimal development of oil and/or gas fields. Haugen (1996) proposed a single parameter representation for uncertainty in the size of reserves and incorporates it into a stochastic dynamic programming model for scheduling of oil fields. However, only decisions related to the scheduling of fields were considered. Meister et al. (1996) presented a model to derive exploration and production strategies for one field under uncertainty in reserves and future oil prices. The model was analyzed using stochastic control techniques. Jonsbraten (1998a) addressed the oilfield development planning problem under oil price uncertainty using an MILP formulation that was solved with a progressive hedging algorithm. Aseeri et al. (2004) introduced uncertainty in the 
oil prices and well productivity indexes, financial risk management, and budgeting constraints into the model proposed by Iyer and Grossmann (1998), and solved the resulting stochastic model using a sampling average approximation algorithm. Jonsbraten (1998b) presented an implicit enumeration algorithm for the sequencing of oil wells under uncertainty in the size and quality of oil reserves. The paper considers investment and operation decisions only for one field. Lund (2000) addressed a stochastic dynamic programming model for evaluating the value of flexibility in offshore development projects under uncertainty in future oil prices and in the reserves of one field using simplified descriptions of the main variables. Cullick et al. (2003) proposed a model based on the integration of a global optimization search algorithm, a finitedifference reservoir simulation, and economics. They presented examples having multiple oil fields with uncertainties in the reservoir volume, fluid quality, deliverability, and costs. Few other papers, (Begg et al., 2001; Zabalza-Mezghani et al., 2004; Bailey et al., 2005; and Cullick et al., 2007), have also used a combination of reservoir modeling, economics and decision making under uncertainty through simulation-optimization frameworks.

However, most of these works either consider very limited flexibility in the investment and operating decisions, or handle the uncertainty in an ad-hoc manner. Stochastic programming provides a systematic framework to model problems that require decision-making in the presence of uncertainty by taking it into account with one or more parameters in terms of probability distribution functions (Birge and Louveaux, 1997). The concept of recourse action in the future, and availability of probability distribution in the context of oilfield development planning problems, makes it one of the most suitable candidates to address uncertainty. Moreover, conservative decisions are usually avoided in the solution utilizing the probability information given the potential of high expected profits in the case of favorable outcomes. In the context of stochastic programming, Goel and Grossmann (2004) considered a gas field development problem under uncertainty in the size and quality of reserves where decisions on the timing of field drilling were assumed to yield an immediate resolution of the uncertainty, i.e. the problem involves decision-dependent uncertainty as discussed in Jonsbraten et al. (1998); Goel and Grossmann (2006); and Gupta and Grossmann (2011). Linear reservoir models, which can provide a reasonable approximation for gas fields, were used. In their solution strategy, the authors used a relaxation problem to predict upper bounds, and solved multistage stochastic programs for a fixed scenario tree for finding lower bounds. Goel et al. (2006) later developed a 
branch and bound algorithm for solving the corresponding disjunctive/mixed-integer programming model where lower bounds were generated by Lagrangean duality. Ettehad et al. (2011) presented a case study for the development planning of an offshore gas field under uncertainty optimizing facility size, well counts, compression power and production policy. Results of two solution methods, optimization with Monte Carlo sampling and stochastic programming were compared, which showed that the stochastic programming approach is more efficient. The models were also used in a value of information (VOI) analysis.

The gradual uncertainty reduction has also been addressed for problems in this class. Stensland and Tjøstheim (1991) have addressed a discrete time problem for finding optimal decisions with uncertainty reduction over time and applied their approach to oil production. These authors expressed the uncertainty in terms of a number of production scenarios. Dias (2002) presented four propositions to characterize technical uncertainty and the concept of revelation towards the true value of the variable. These four propositions, based on the theory of conditional expectations, are employed to model technical uncertainty. Tarhan et al. (2009) addressed the planning of offshore oil field infrastructure involving endogenous uncertainty in the initial maximum oil flowrate, recoverable oil volume, and water breakthrough time of the reservoir, where decisions affect the resolution of these uncertainties in a gradual manner. The authors developed a multistage stochastic programming model and duality-based branch and bound algorithm taking advantage of the problem structure and globally optimizing each scenario problem independently. An improved solution approach was also proposed that combines global optimization and outer-approximation to optimize the investment and operations decisions (Tarhan et al., 2011). However, the model considers either gas/water or oil/water components for single field and single reservoir at a detailed level. Hence, realistic multi-field site instances can be expensive to solve with this model.

It should be noted that there are multiple sources of uncertainty in the oil and gas field development problem as can be seen from the afore-mentioned literature work. The market price of oil/gas, quantity and quality of reserves at a field are the most important sources of the uncertainty in this context. The uncertainty in oil prices is influenced by the political, economic or other market factors and it belongs to the exogenous uncertainty problems. The uncertainty in the reserves on the other hand, is linked to the accuracy of the reservoir data (technical uncertainty). While the existence of oil and gas at a field is indicated by seismic surveys and 
preliminary exploratory tests, the actual amount of oil in a field, and the efficiency of extracting the oil will only be known after capital investment has been made at the field (Goel and Grossmann, 2004), i.e. endogenous uncertainty. Both, the price of oil and the quality of reserves directly affect the overall profitability of a project, and hence it is important to consider the impact of these uncertainties when formulating the decision policy. However, due to the significant computational challenge in this paper we only address the uncertainty in the field parameters where timing of uncertainty realizations is decision-dependent. In particular, we focus on the type of endogenous uncertainty where the decisions are used to gain more information, and resolve uncertainty either immediately or in a gradual manner. Therefore, the resulting scenario tree is decision-dependent that requires modeling a superstructure of all possible scenario trees that can occur based on the timing of the decisions (see Goel et al., 2006; Tarhan et al., 2009).

In this paper, we present a general multistage stochastic programming model for multiperiod investment and operations planning of offshore oil and gas field infrastructure. The model considers the deterministic models proposed in Gupta and Grossmann (2012a, 2012b) as a basis to extend to the stochastic programming using the modeling framework presented in Gupta and Grossmann (2011) for endogenous (decision-dependent) uncertainty problems. In terms of the fiscal contracts, we consider progressive production sharing agreements, whereas the endogenous uncertainty in the field parameters i.e. field size, oil deliverability, water-oil ratio and gas-oil ratio is considered, that can only be revealed once an investment is made in the field and production is started in it. Compared to the conventional models where either fiscal rules or uncertainty in the field parameters are taken into account, the proposed model is the first one in the literature that also allows considering both of these complexities simultaneously. To solve large instances of the problem, the Lagrangean decomposition approach similar to Gupta and Grossmann (2011), allowing parallel solution of the scenario subproblems, is implemented in the GAMS grid computing environment.

The outline of this paper is as follows. First, we present a detailed problem description for offshore oilfield development planning under production sharing agreements and endogenous uncertainties. The corresponding multistage stochastic programming model is presented next in extensive as well as in compact form. The Lagrangean decomposition algorithm adapted from Gupta and Grossmann (2011) is explained to solve the stochastic oilfield planning model. The 
proposed model and solution approach are then applied to multiple instances of the two oilfield development problems to illustrate their performances.

\section{Problem Statement}

In this paper, we consider the development planning of an offshore oil and gas field infrastructure under complex fiscal rules and endogenous uncertainties. In particular, a multifield site, $F=\{1,2, \ldots\}$, with potential investments in floating production storage and offloading (FPSO) facilities, FPSO $=\{1,2, \ldots\}$ with continuous capacities and ability to expand them in the future is considered (Figure 1). The connection of a field to an installed FPSO facility and a number of wells need to be drilled to produce oil from these fields for the given planning horizon. The planning horizon is discretized into T time periods, typically each with one year duration. The location of each FPSO facility and its possible connections to the given fields are assumed to be known. Notice that each FPSO facility can be connected to more than one field to produce oil, while a field can only be connected to a single FPSO facility due to engineering requirements and economic viability of the project. For simplicity, we only consider FPSO facilities. The proposed model can easily be extended to other facilities such as tension leg platforms (TLPs). The water produced with the oil is usually re-injected after separation, while the gas can be sold in the market. In this case, we consider natural depletion of the reserves, i.e. no water or gas re-injection.

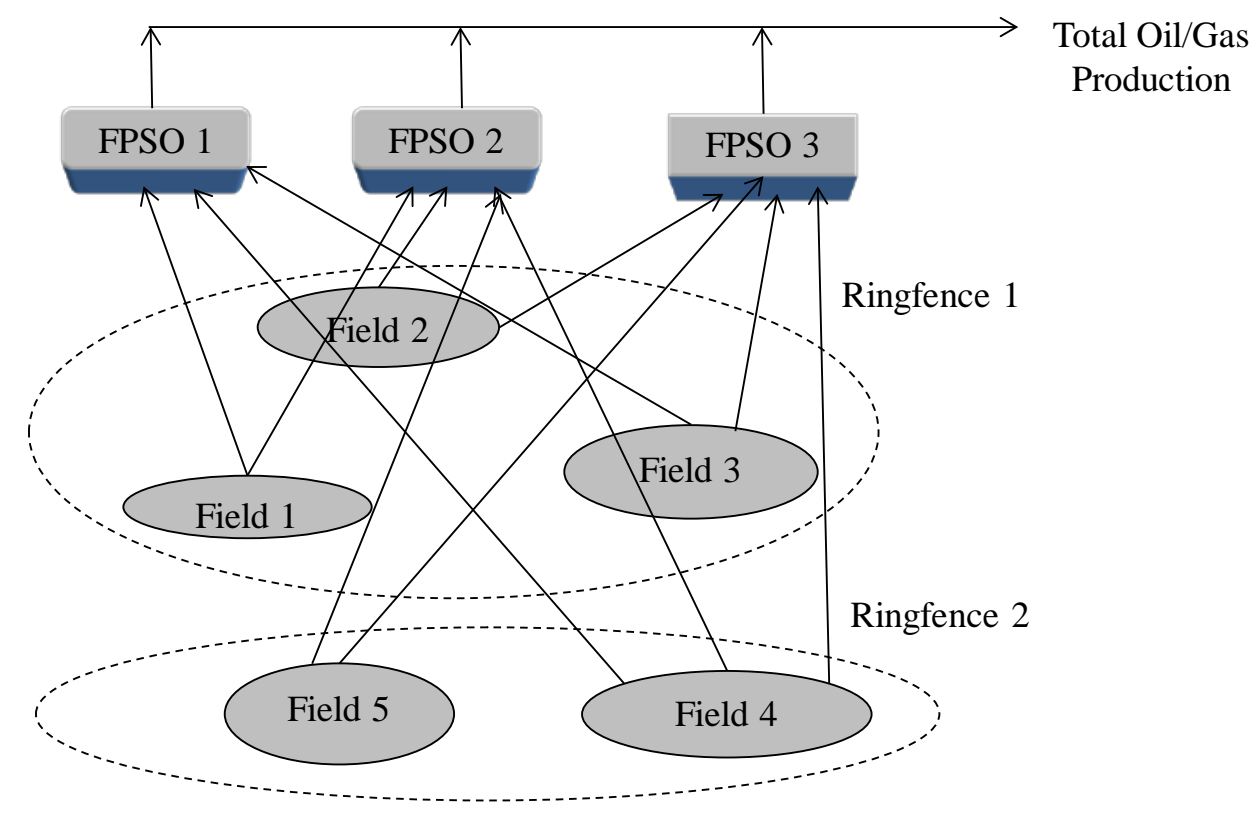

Figure 1: A typical offshore oilfield infrastructure representation 
There are three major complexities in the problem considered here:

2.1 Nonlinear Reservoir Profiles: We consider three components (oil, water and gas) explicitly during production from a field. Field deliverability, i.e. maximum oil flowrate from a field, water-oil-ratio (WOR) and gas-oil-ratio (GOR) are approximated by cubic equations (a)-(c) (see Figure 2), while cumulative water produced and cumulative gas produced from a field are represented by fourth order separable polynomials, eqs. (d)-(e) (see Gupta and Grossmann, 2012a for details).

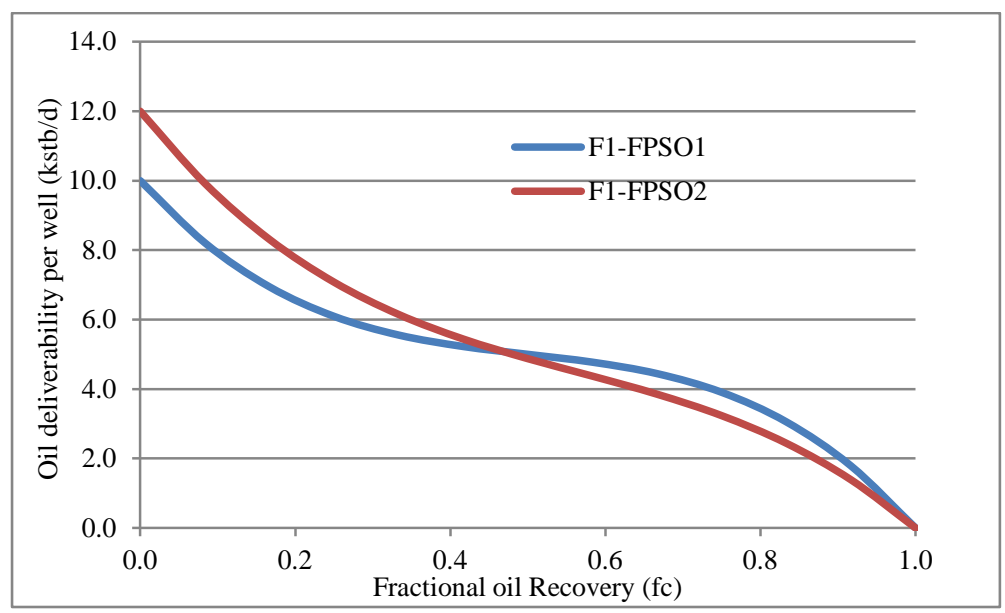

(a) Oil Deliverability per well for field (F1)

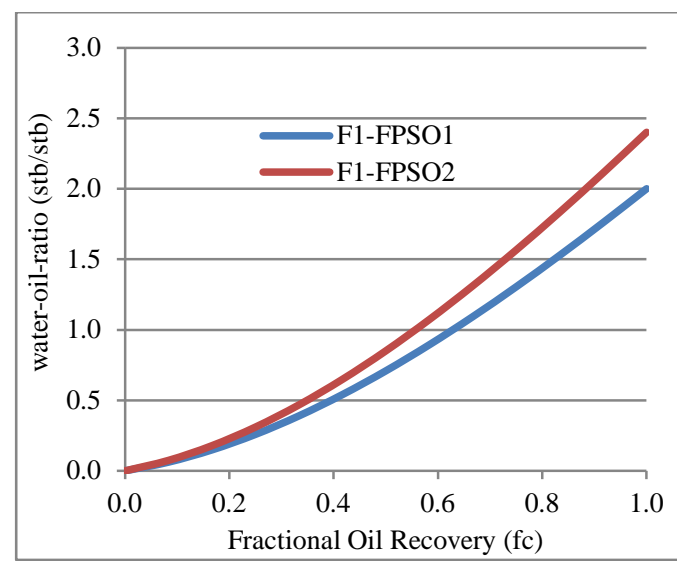

(b) Water to oil ratio for field (F1)

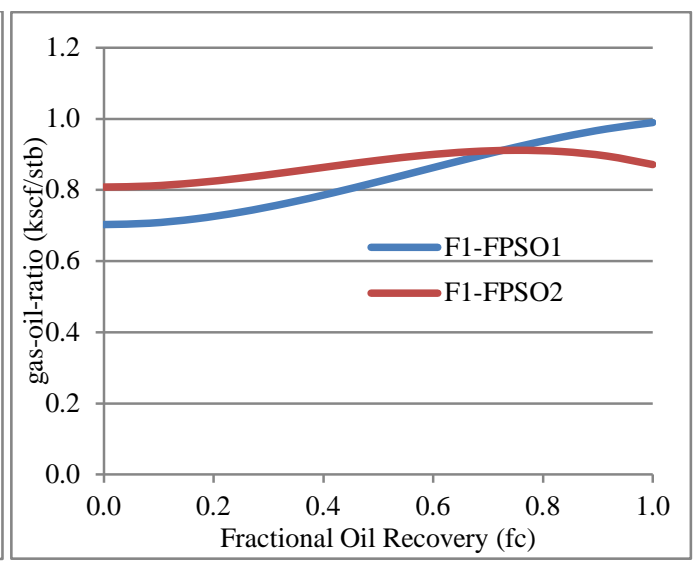

(c) Gas to oil ratio for field (F1)

Figure 2: Nonlinear Reservoir Characteristics for field (F1) for 2 FPSOs (FPSO 1 and 2)

$$
\begin{array}{lll}
\hat{Q}_{f}^{d}=a_{1, f}\left(f c_{f, t}\right)^{3}+b_{1, f}\left(f c_{f}\right)^{2}+c_{1, f} f c_{f}+d_{1} & \forall f & \text { (a) } \\
\text { wôr } r_{f}=a_{2, f}\left(f c_{f}\right)^{3}+b_{2, f}\left(f c_{f}\right)^{2}+c_{2, f} f c_{f}+d_{2, f} & \forall f & \text { (b) } \\
g \hat{o} r_{f}=a_{3, f}\left(f c_{f}\right)^{3}+b_{3, f}\left(f c_{f}\right)^{2}+c_{3, f} f c_{f}+d_{3, f} & \forall f & \text { (c) }
\end{array}
$$




$$
\begin{array}{lll}
\hat{w} c_{f}=a_{4, f}\left(f c_{f}\right)^{4}+b_{4, f}\left(f c_{f}\right)^{3}+c_{4, f} f c_{f}^{2}+d_{4, f} f c_{f} & \forall f & \text { (d) } \\
\hat{g} c_{f}=a_{5, f}\left(f c_{f}\right)^{4}+b_{5, f}\left(f c_{f}\right)^{3}+c_{5, f} f c_{f}^{2}+d_{5, f} f c_{f} & \forall f & \text { (e) } \\
w_{f}=w o \hat{r} r_{f} \cdot x_{f} & \forall f & (\mathrm{f}) \\
g_{f}=g \hat{o} r_{f} \cdot x_{f} & \forall f & \text { (g) }
\end{array}
$$

The motivation for using the polynomials for cumulative water produced and cumulative gas produced in eqs. (d)-(e) as compared to WOR and GOR in eqs. (b)-(c) is to avoid bilinear terms, eqs. (f)-(g), in the formulation and allow converting the resulting model into an MILP formulation using piecewise linear approximations. All the wells in a particular field $f$ are assumed to be identical for the sake of simplicity leading to the same reservoir profiles, eqs. (a)(g), for each of these wells.

2.2 Production Sharing Agreements: There are fiscal contracts with the host government that need to be accounted for during development planning. In particular, we consider progressive (sliding scale) production sharing agreements with ringfencing provisions, which are widely used in several countries. The revenue flow in a typical production sharing agreement (PSA) can be seen as in Figure 3 (World Bank, 2007). First, in most cases, the company pays royalty to the government at a certain percentage of the total oil produced. After paying the royalties, some portion of the remaining oil is treated as cost oil by the oil company to recover its costs. There is a ceiling on the cost oil recovery to ensure revenues to the government as soon as production starts. The remaining part of the oil, called profit oil, is divided between oil company and the host government at a certain percentage. The oil company needs to further pay income tax on its share of profit oil. Hence, the total contractor's (oil company) share in the gross revenue is comprised of cost oil and contractor's profit oil share after tax.

In this work, we consider a sliding scale profit oil share of the contractor linked to the cumulative oil produced. For instance, if the cumulative production (in MMbbl) is in the range of first tier, $0 \leq x c_{t} \leq 200$, the contractor receives $50 \%$ of the profit oil, while if the cumulative production (in MMbbl) reaches tier 2, 200 $\leq x c_{t} \leq 400$, the contractor receives $40 \%$ of the profit oil, and so on (see Figure 4). Notice that this tier structure is a step function, which requires additional binary variables to model and makes the problem harder to solve. Moreover, the cost recovery ceiling is considered to be a fraction of the gross revenues in each time period t. For 
simplicity, the cost recovery ceiling fraction and income tax rates are assumed to be fixed percentages (no sliding scale), and there are no explicit royalty provisions which is a straightforward extension.

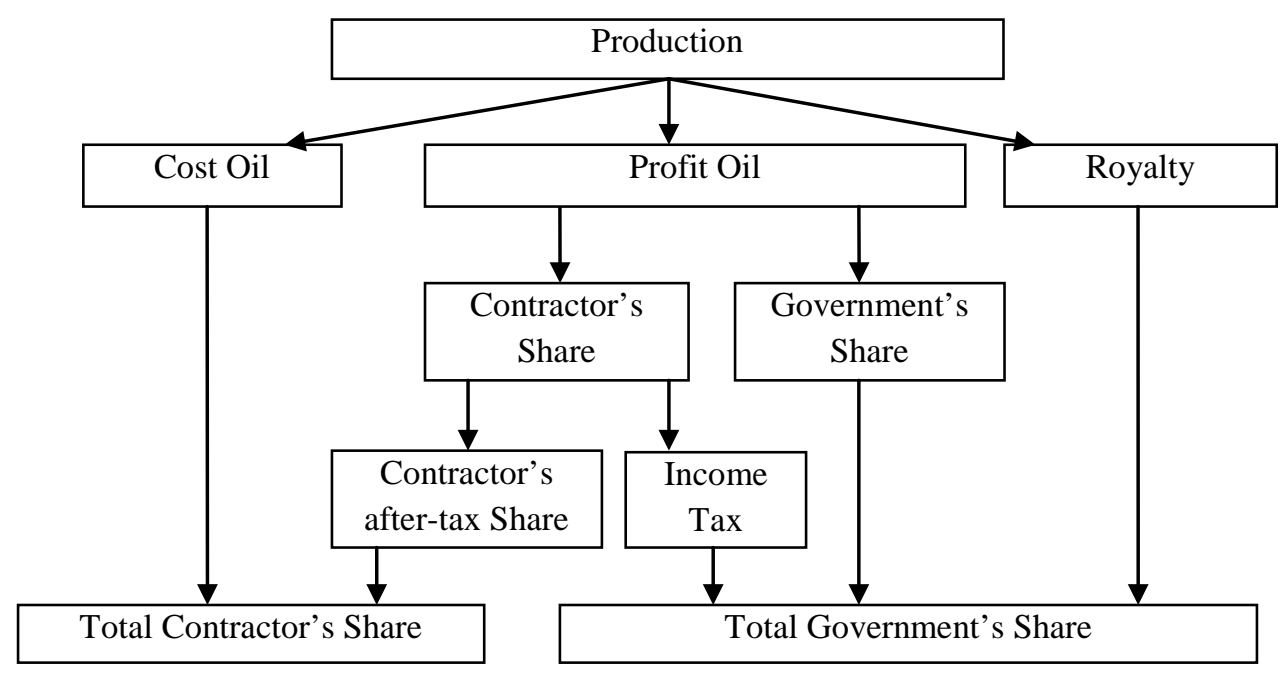

Figure 3: Revenue flow for a typical Production Sharing Agreement

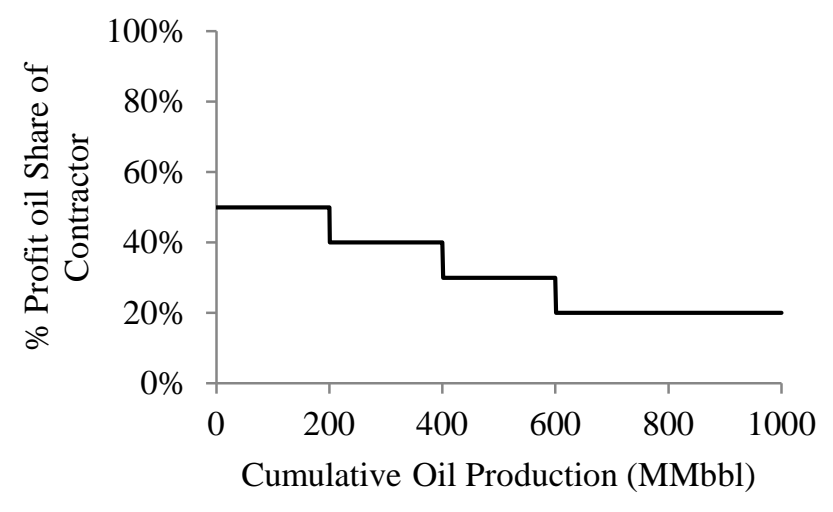

Figure 4: Progressive profit oil share of the contractor

A set of ringfences $R F=\{1,2, \ldots\}$ among the given fields is specified (see Figure 1) to ensure that fiscal calculations are applied for each ringfence separately (see Gupta and Grossmann (2012b) for details). For example, the fiscal calculations for Fields 1-3 (Ringfence 1) and Field 4-5 (Ringfence 2) in Figure 1 cannot be consolidated in one place. These ringfences may or may not have the same fiscal rules. Qualitatively, a typical ringfencing provision states that the investment and operational costs for a specified group of fields or block can only be recovered from the revenue generated from those fields or block. Notice that in general a field is 
associated to a single ringfence, while a ringfence can include more than one field. In contrast, a facility can be connected to multiple fields from different ringfences for producing oil and gas.

\subsection{Endogenous Uncertainties:}

(a) Uncertain Field Parameters: We consider here the uncertainty in the field parameters, i.e. field size, oil deliverability per well, water-oil ratio and gas-oil ratio. These are endogenous uncertain parameters since investment and operating decisions affect the stochastic process (Jonsbraten et al., 1998; Goel et al., 2006; Tarhan et al., 2009; and Gupta and Grossmann, 2011). In particular, the uncertainty in the field parameters can only be resolved when an investment is made in that field and production is started in it. Therefore, optimization decisions determine the timing of uncertainty realization, i.e. decision-dependent uncertainty (type 2).

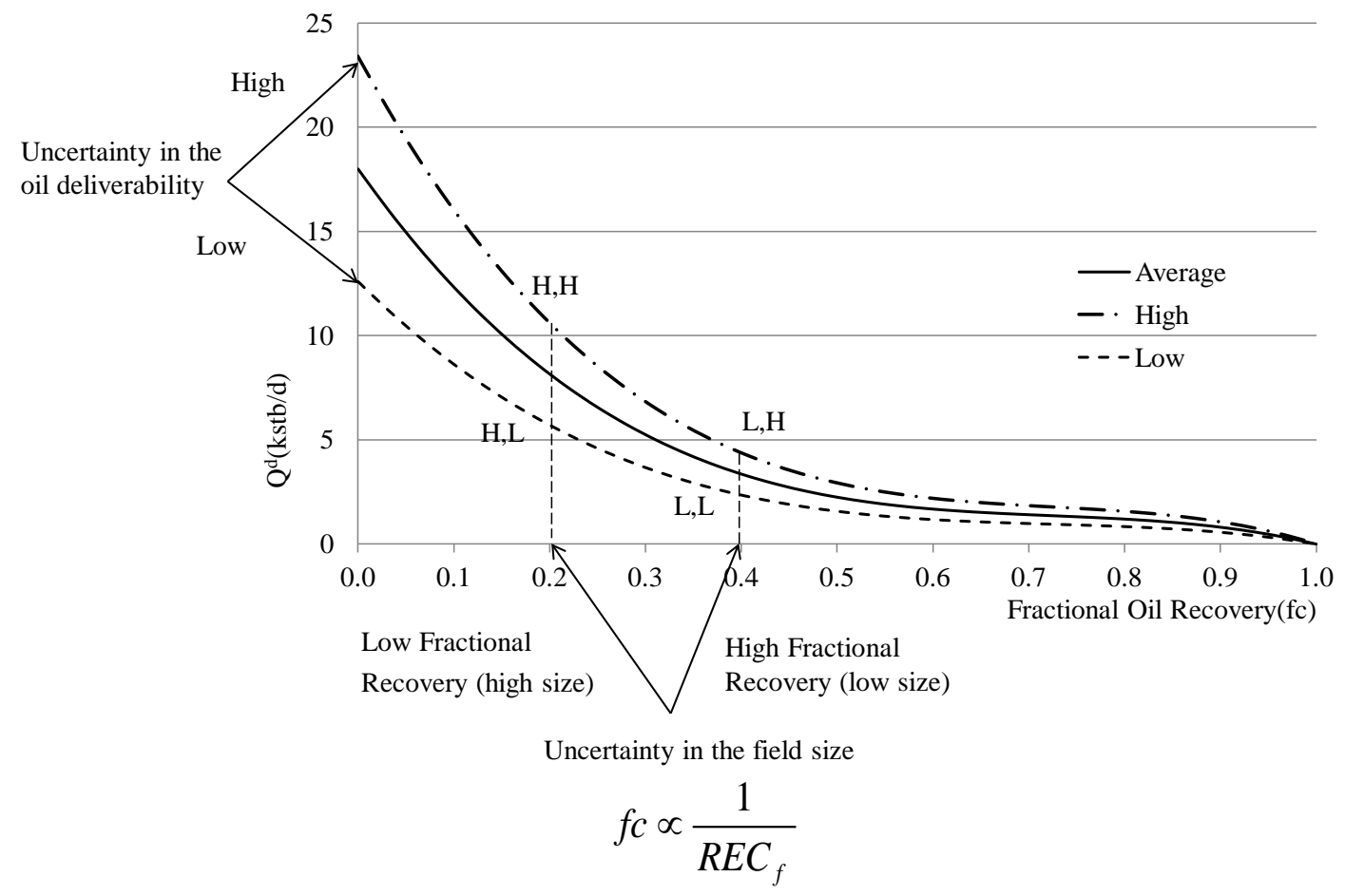

Figure 5: Oil deliverability per well for a field under uncertainty

The average profile in Figure 5 represents the oil deliverability per well for a field as a nonlinear polynomial in terms of the fractional oil recovery (eq. (a)) under perfect information. However, due to the uncertainty in the oil deliverability, the actual profile is assumed to be either the lower or upper side of the average profile with a given probability. In particular, eq. (h) represents the oil deliverability per well for a field under uncertainty where parameter $\alpha_{f, \text { oil }}$ is used to characterize this uncertainty. 


$$
Q_{f}^{d}=\alpha_{f, o i l} \cdot \hat{Q}_{f}^{d} \quad \forall f
$$

For instance, if $\alpha_{f, \text { oil }}>1$, then we have a higher oil deliverability than expected $\left(\alpha_{f, \text { oil }}=1\right)$, whereas for $\alpha_{f, \text { oil }}<1$ a lower than expected oil deliverability is observed. Since, the uncertain field size (recoverable oil volume, $R E C_{f}$ ) is an inverse function of the fraction oil recovery, a higher field size will correspond to the low fractional oil recovery, whereas a small field size will correspond to the higher fractional oil recovery for a given amount of the cumulative oil production.

Similarly, eqs. (i) and (j) correspond to the uncertain field profiles for water-oil-ratio and gas-oil-ratio that are characterized by the uncertain parameters $\alpha_{f, \text { wor }}$ and $\alpha_{f, \text { gor }}$, respectively. Notice that since the cumulative water produced (eq. (d)) and the cumulative gas produced (eq. (e)) profiles are used in the model, instead of water-oil-ratio (eq. (b)) and gas-oil-ratio (eq. (c)), the uncertainty in the parameters $\alpha_{f \text {,wor }}$ and $\alpha_{f, g o r}$ can be transformed into the corresponding uncertainty in the parameters $\alpha_{f, w c}$ and $\alpha_{f, g c}$ as in eqs. (k) and (l), respectively. In particular, we use the correspondence among the coefficients of these two sets of the polynomials for this transformation (Gupta and Grossmann, 2012a).

$$
\begin{array}{lc}
w_{f o r_{f}}=\alpha_{f, w o r} \cdot w \hat{o r} & \forall f \\
g o r_{f}=\alpha_{f, g o r} \cdot g \hat{o} r_{f} & \forall f \\
w c_{f}=\alpha_{f, w c} \cdot \hat{w} c_{f} & \forall f \\
g c_{f}=\alpha_{f, g c} \cdot \hat{g} c_{f} & \forall f
\end{array}
$$

Moreover, the uncertain parameters for every field, i.e. $\theta_{f}=\left\{R E C_{f}, \alpha_{f, \text { oil }}, \alpha_{f, \text { wor }}, \alpha_{f, \text { gor }}\right\}$ are considered to have a number of possible discrete realizations $\tilde{\theta}_{f}^{k}$ with a given probability. Therefore, all the possible combinations of these realizations yield a set of scenarios $s \in S^{\text {sup }}$ where each scenario has the corresponding probability $p^{s}$.

(b) Correlation among the uncertain parameters: If the uncertain parameters are considered to be independent, the total number of scenarios defined by the set $S^{\text {sup }}$ grows exponentially with the number of uncertain parameters and their possible realizations, which makes the problem 
intractable. For instance, if there are only 2 fields, then 4 uncertain parameters for each field having 2 realizations will require 256 scenarios. Therefore, it becomes difficult to solve a multifield site problem with independent uncertainties in the set $\theta_{f}=\left\{R E C_{f}, \alpha_{f, \text { oil }}, \alpha_{f, \text { wor }}, \alpha_{f, \text { gor }}\right\}$. Since in practice normally uncertainties are not independent, we can overcome this limitation by considering that there are correlations among the uncertain parameters for each individual field. In particular, the uncertain parameters for a field $\theta_{f}=\left\{R E C_{f}, \alpha_{f, \text { oil }}, \alpha_{f, \text { wor }}, \alpha_{f, \text { gor }}\right\}$ are considered to be dependent. Therefore, only a subset of the possible scenarios $S \subset S^{\text {sup }}$ is sufficient to represent the uncertainty. For instance, based on practical considerations, we can assume that if a field is of lower size than expected, then the oil deliverability is also lower $\left(\alpha_{f, \text { oil }}<1\right)$. Therefore, the scenarios with a combinations of higher oil deliverability $\left(\alpha_{f, \text { oil }}>1\right)$ and lower field size are not included in the reduced scenario set and vice-versa. Similarly, correlations for the water-oil ratio and gas-oil ratio can be considered to substantially reduce the original scenario set $S^{\text {sup }}$. Therefore, the problem can be considered as selecting a sample of the scenarios for each field, where a scenario for that field will be equivalent to the selected combinations of the realizations of the uncertain parameters $\theta_{f}=\left\{R E C_{f}, \alpha_{f, \text { oil }}, \alpha_{f, \text { wor }}, \alpha_{f, \text { gor }}\right\}$.

In the computational experiments, we only consider the extreme cases of the scenarios assuming perfect correlations, i.e. all uncertain parameters for a field have either low, medium or high realizations. Note that these assumptions on correlation among the field parameters are flexible and can be modified depending on the problem at hand. In addition to the correlation among the uncertain parameters for each individual field, one can also take into account the correlation among the fields based on the available information for a particular oilfield development site to further reduce the total number of scenarios. Notice also that the model and solution method presented in the paper is irrespective of whether a reduced scenario set $S$ is considered or the complete one ( $S^{\text {sup }}$ ).

(c) Uncertainty Resolution Rules: Instead of assuming that the uncertainties are resolved as soon as a well is drilled in the field, i.e. immediate resolution, we assume that several wells need to be drilled and production has to be started from the field for this purpose. Moreover, since the uncertain parameters for a field $\theta_{f}=\left\{R E C_{f}, \alpha_{f, \text { oil }}, \alpha_{f, \text { wor }}, \alpha_{f, g o r}\right\}$ are assumed to be correlated as described above, the timing of uncertainty resolution in these parameters is also considered to the 
same. This allows solving much larger multi-field site instances without losing much in terms of the quality of the solution.

In contrast, Tarhan et al. (2009) considered a single field at a detailed level where no correlations among the uncertain parameters of the field were considered, and these parameters were allowed to be revealed independently at different time periods in the planning horizon. However, the resulting scenario tree even for a single field becomes very complex to model and solve. Therefore, we assume that the uncertainty in all the field parameters $\theta_{f}=\left\{R E C_{f}, \alpha_{f, \text { oil }}, \alpha_{f, \text { wor }}, \alpha_{f, \text { gor }}\right\}$ are resolved if at-least $\mathrm{N}_{1}$ number of wells have been drilled in the field, and production has been performed from that field for a duration of at-least $\mathrm{N}_{2}$ years. Notice that these assumptions on uncertainty resolution rules are flexible and can be adapted depending on the field information that is available. Moreover, the model can also be extended to the case where each parameter for a field is allowed to be revealed in different years based on the work of Tarhan et al. (2009) that will result in a significant increase in the computation expense.

(d) Decision-dependent scenario trees: The multiperiod planning horizon and the discrete set of the selected scenarios for each field with given probabilities can be represented by scenario trees. However, since the timing of the uncertainty realization for a field (or its corresponding scenarios) depends on the drilling and operating decisions, the resulting scenario tree is also decision-dependent (see Gupta and Grossmann, (2011) for details). For instance, if we consider a set of two uncertain fields $F=\{1,2\}$ and the selected scenario set based on the parameter correlations for each field has 2 elements, $\left\{\tilde{\theta}_{f}^{1}, \tilde{\theta}_{f}^{2}\right\}$, with equal probability. Therefore, the problem involves the following 4 scenarios each with a probability of 0.25 :

$$
S=\left\{1:\left(\tilde{\theta}_{1}^{1}, \tilde{\theta}_{2}^{1}\right) ; 2:\left(\tilde{\theta}_{1}^{1}, \tilde{\theta}_{2}^{2}\right) ; 3:\left(\tilde{\theta}_{1}^{2}, \tilde{\theta}_{2}^{1}\right) ; 4:\left(\tilde{\theta}_{1}^{2}, \tilde{\theta}_{2}^{2}\right)\right\}
$$

Notice that each of these elements, $\left\{\tilde{\theta}_{f}^{1}, \tilde{\theta}_{f}^{2}\right\}$, is equivalent to a selected combination of the realization of the corresponding uncertain parameters, for example $\tilde{\theta}_{f}^{1}=\left\{R E C_{f}^{1}, \alpha_{f, \text { oil }}^{1}, \alpha_{f, \text { wor }}^{1}, \alpha_{f, \text { oor }}^{1}\right\}$. Figure 6 represents the scenario tree for this problem, where the uncertainty in the first field is resolved at the end of first year, since we drill $\mathrm{N}_{1}$ wells in the field at the beginning of year 1 and produce from this field during that year $\left(\mathrm{N}_{2}=1\right)$. The system can be in two different states in year 2 depending on the realized value of the uncertain parameter $\tilde{\theta}_{1}^{k}$. Similarly, uncertainty in the field 2 is resolved in year 4 under the scenarios 3 and 
4 due to drilling and operating decisions, whereas it remains uncertain in the scenarios 1 and 2. Therefore, the resulting scenario tree depends on the optimization decisions, which are not known a priori, requiring modeling a superstructure of the all possible scenario trees that can occur based on our decisions. Notice that the scenario-tree also allows considering the cases where the number of wells drilled in a field is less than the one required for the uncertainty resolution (i.e. $\mathrm{N}_{1}$ wells), and therefore, the corresponding scenarios remain indistinguishable.

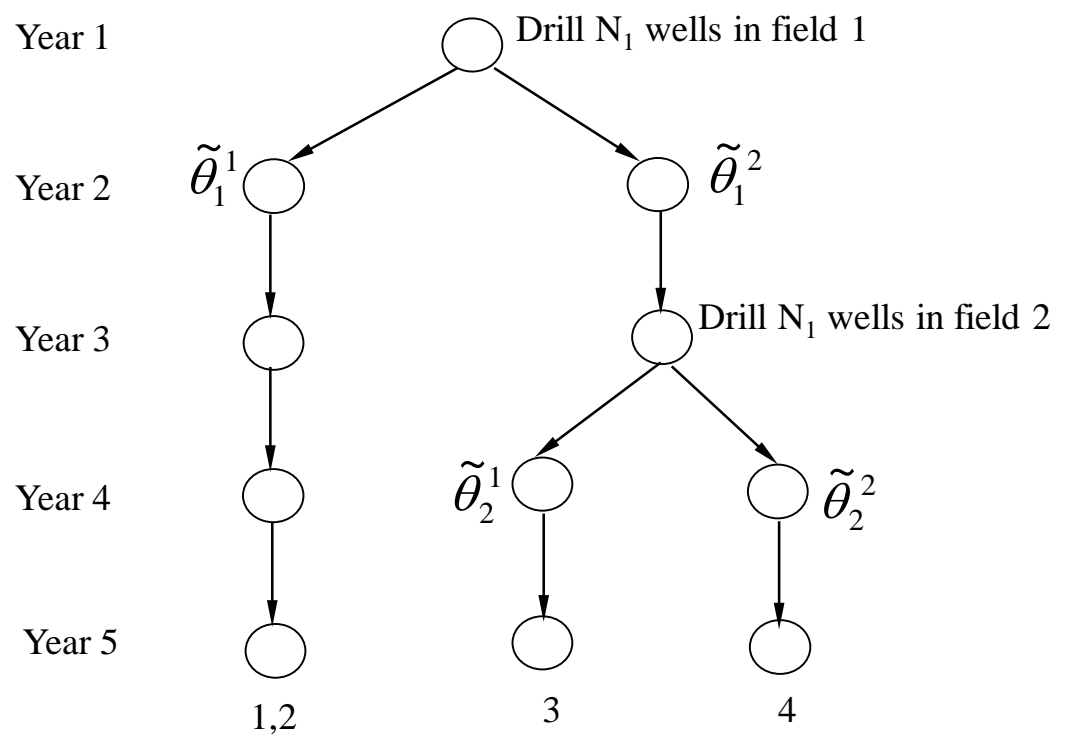

Figure 6: Decision-dependent scenario tree for two fields

An alternate representation of the decision-dependent scenario-tree (Ruszczynski, 1997) is used to model the problem as a multistage stochastic program in which the scenarios are treated independently and related through the non-anticipativity constraints for states of different scenarios that are identical (see Goel and Grossmann, 2006; and Gupta and Grossmann, 2011).

The problem is to determine the optimal investment and operating decisions to maximize the contractor's expected NPV for a given planning horizon considering the above production sharing agreements and endogenous uncertainties. In particular, investment decisions in each time period $t$ and scenario $s$ include FPSO facilities installation or expansion, and their respective installation or expansion capacities for oil, liquid and gas, fields-FPSO connections, and the number of wells that need to be drilled in each field $f$, given the restrictions on the total number of wells that can be drilled in each time period $t$ over all the given fields. Operating decisions include the oil/gas production rates from each field $f$ in each time period $t$ under every scenario s. 
It is assumed that the installation and expansion decisions occur at the beginning of each time period $t$, while operations take place throughout the time period. There is a lead time of $l_{1}$ years for each FPSO facility initial installation, and a lead time of $l_{2}$ years for the expansion of an earlier installed FPSO facility. Once installed, we assume that the oil, liquid (oil and water) and gas capacities of a FPSO facility can only be expanded once. These assumptions are made for the sake of simplicity, and both the model and the solution approaches are flexible enough to incorporate more complexities. In the next section, we propose a multistage stochastic programming model for oilfield development planning with production sharing agreements and decision-dependent uncertainty in the field parameters as described.

\section{Multistage Stochastic Programming Model}

We present a general multistage stochastic programming model for offshore oilfield development planning that considers the trade-offs involved between investment and operating decisions, uncertainties in the field parameters and profit share with the government while maximizing the overall expected NPV for the contractor. Notice that the model is intended to be applied every year of the project in a rolling horizon manner, not just once for the entire planning horizon. In this way, new data for the model is updated as it becomes available every year. The constraints involved in the model are as follows:

(i) Objective Function: The objective function is to maximize the total expected NPV of the contractor as in (1), which is the summation of the NPVs over all the scenarios having probabilities $p^{s}$. The NPV of a particular scenario $s$ is the difference between discounted total contractor's gross revenue share and total cost over the planning horizon (2). The total contractor's share in a particular time period t and scenario s is the sum of the contractor's share over all the ring-fences ( $r f$ ) as given in equation (3). Similarly, constraints (4) and (5) represent the total capital and operating expenses for each scenario $s$ in time period $t$.

Max ENPV

$E N P V=\sum_{s} p^{s} \sum_{t} d i s_{t} \cdot\left(\right.$ TotalConSh tot,s $\left.-C A P_{t}^{\text {tot }, s}-O P E R_{t}^{\text {tot }, s}\right)$

TotalConSh $_{t}^{\text {tot,s }}=\sum_{r f}$ TotalConSh $h_{r f, t}^{s} \quad \forall t, s$ 


$$
\begin{array}{ll}
C A P_{t}^{\text {tot,s }}=\sum_{r f} C A P_{r f, t}^{s} & \forall t, s \\
O P E R_{t}^{\text {tot, } s}=\sum_{r f} O P E R_{r f, t}^{s} & \forall t, s
\end{array}
$$

(ii) Cost Calculations: The total capital expenses in scenario s for a ring-fence $r f$ contains two components as given in equation (6). One is field specific (eq. 7) that accounts for the connection costs between a field and a FPSO facility, and cost of drilling the wells for each of the field in that ring-fence $r f$. The other cost component is FPSO specific (eq. 8) that includes the capital expenses for the corresponding FPSO facilities. Eq. (9) calculates the total cost of an FPSO facility in time period t for scenario s which is disaggregated in eq. (10) over various fields (and therefore ring-fences as in (11)). The cost disaggregation is performed on the basis of the field sizes to which the FPSO is connected (eq. (12)-(14)), where set $F_{\text {fpso }}$ is the set of all the fields that can be connected to FPSO facility fpso and the binary variable $b_{f, \text { fpso }}^{o n, s}$ represents the potential connections. Notice that there is an uncertainty in the recoverable oil volume of the field $\left(R E C_{f}^{s}\right)$ used in eq. (14) that multiplies the binary variable $b_{f, \text { opso }}^{\text {on,s }}$. To linearize the bilinear terms in eq. (14), we use an exact linearization technique (Glover, 1975) by introducing the positive variables $\left(Z D_{f^{\prime}, f, f p s o, t}^{\text {field, }}, Z D 1_{f^{\prime}, f, f \text { fies }, t}^{\text {fiet },}\right)$ and $\left(Z D_{f, f p s o, t}^{s}, Z D 1_{f, f p s o, t}^{s}\right)$ that results in the constraints (15)-(23).

$$
\begin{array}{ll}
C A P_{r f, t}^{s}=C A P 1_{r f, t}^{s}+C A P 2_{r f, t}^{s} & \forall r f, t, s \\
C A P 1_{r f, t}^{s}=\sum_{F_{r f}} \sum_{f p s o} F C_{f, f p s o, t} b_{f, f p s o, t}^{s}+\sum_{F_{r f}} F C_{f, t}^{\text {well }} I_{f, t}^{\text {well,s }} & \forall r f, t, s \\
C A P 2_{r f, t}^{s}=\sum_{f p s o} D F P S O C_{r f, f p s o, t}^{s} & \forall r f, t, s \\
F P S O C_{f p s o, t}^{s}=\left[F C_{f p s o, t}^{F P S O} F_{f p s o, t}^{F P S O, s}+V C_{f p s o, t}^{l i q}\left(Q I_{f p p s o, t}^{l i q, s}+Q E_{f p s o, t}^{l i q, s}\right)\right. & \left.V C_{f p s o, t}^{g a s}\left(Q I_{f p s o, t}^{g a s, s}+Q E_{f p s o, t}^{g a s, s}\right)\right] \\
F P S O C_{f p s o, t}^{s}=\sum_{F_{f p s o}} D F P S O C_{f, f p s o, t}^{f i e l d, s} & \forall r f, t, s \\
D F P S O C_{r f, f p s o, t}^{s}=\sum_{F_{r f}} D F P S O C_{f, f p s o, t}^{f i e l d, s} & \forall r f, t, s
\end{array}
$$




$$
\begin{aligned}
& b_{f, f p s o}^{o n, s}=\sum_{t} b_{f, f p s o, t}^{s} \quad \forall f, f p s o, s \\
& \operatorname{DFPSOC}_{f, f p s o, t}^{f i e l d, s} \leq M \cdot b_{f, f p s o}^{o n, s} \quad \forall f, f p s o, t, s \\
& \operatorname{DFPSOC}_{f, f p s o, t}^{f i e l d, s}=\frac{b_{f, f p s o}^{o n, s} \cdot R E C_{f}^{s}}{\sum_{f^{\prime} \in F_{\text {fpso }}} b_{f^{\prime}, \text { fpso }}^{\text {on }} \cdot R E C_{f^{\prime}}^{s}} \cdot \text { FPSOC }_{f p s o, t}^{s} \quad \forall f, f p s o, t, s \\
& \sum_{f^{\prime} \in F_{f p s o}} Z D_{f^{\prime}, f, f p s o, t}^{f i e l d, s} \cdot R E C_{f^{\prime}}^{s}=Z D_{f, f p s o, t}^{s} \cdot R E C_{f}^{s} \quad \forall f, \text { fpso, } t, s \\
& Z D_{f^{\prime}, f, f p s o, t}^{f i e l d, s}+Z D 1_{f^{\prime}, f, f p s o, t}^{\text {field } s}=D F P S O C_{f, f p s o, t}^{f i e l d, s} \quad \forall f, f p s o, t, f^{\prime} \in F_{f p s o}, s \\
& Z D_{f^{\prime}, f, f p s o, t}^{\text {field }, s} \leq U \cdot b_{f^{\prime}, \text { fpso }}^{o n, s} \quad \forall f, \text { fpso, } t, f^{\prime} \in F_{f p s o}, s \\
& Z D 1_{f^{\prime}, f, f p s o, t}^{\text {field,s }} \leq U \cdot\left(1-b_{f^{\prime}, f p s o}^{o n, s}\right) \quad \forall f, f p s o, t, f^{\prime} \in F_{f p s o}, s \\
& Z D_{f^{\prime}, f, f p s o, t}^{\text {field }, s} \geq 0, Z D 1_{f^{\prime}, f, f p s o, t}^{\text {field }, s} \geq 0 \quad \forall f, f p s o, t, f^{\prime} \in F_{f p s o}, s \\
& Z D_{f, f p s o, t}^{s}+Z D 1_{f, f p s o, t}^{s}=F_{P S O C}^{s} \quad \forall f, f p s o, t, s \\
& Z D_{f, f p s o, t}^{s} \leq U \cdot b_{f, f p s o}^{o n, s} \quad \forall f, f p s o, t, s \\
& Z D 1_{f, f p s o, t}^{s} \leq U \cdot\left(1-b_{f, f p s o}^{o n, s}\right) \quad \forall f, f p s o, t, s \\
& Z D_{f, f p s o, t}^{s} \geq 0, Z D 1_{f, f p s o, t}^{s} \geq 0 \quad \forall f, f p s o, t, s
\end{aligned}
$$

The total operating expenses for scenario s in time period t for ring-fence $r f$, eq. (24), are the operation costs corresponding to the total amount of liquid and gas produced.

$$
O P E R_{r f, t}^{s}=\delta_{t}\left[O C_{r f, t}^{l i q}\left(x_{r f, t}^{t o t, s}+w_{r f, t}^{t o t, s}\right)+O C_{r f, t}^{g a s} g_{r f, t}^{t o t, s}\right] \quad \forall r f, t, s
$$

(iii) Total Contractor Share Calculations: The total contractor share in scenario s for ringfence $r f$ in time period t, eq. (25), is the sum of contractor's after-tax profit oil share for that ringfence and the cost oil that it keeps to recover the expenses. The contractor's profit oil share after tax in scenario $s$ is the difference of the contractor's profit oil share before tax and income tax 
paid as in constraint (26). The tax paid by the contractor on its profit oil share depends on the given tax rate $\left(f_{r f, t}^{t a x}\right)$ as in constraint (27).

$$
\begin{array}{ll}
\text { TotalConSh }_{r f, t}^{s}=\text { ConSh }_{r f, t}^{\text {aftertax }, s}+\text { CO }_{r f, t}^{s} & \forall r f, t, s \\
\text { ConSh }_{r f, t}^{\text {aftertax }, s}=\text { ConSh }_{r f, t}^{\text {beforetax }, s}-\operatorname{Tax}_{r f, t}^{s} & \forall r f, t, s \\
\operatorname{Tax}_{r f, t}^{s}=f_{r f, t}^{\text {tax }} \cdot \text { ConSh }_{r f, t}^{\text {beforetax }, s} & \forall r f, t, s
\end{array}
$$

The contractor's share before tax for scenario s in each time period $t$ is some fraction of the total profit oil during that period t for ring-fence $r f$. Note that we assume here that this profit oil fraction, $f_{r f, i}^{p o}$, is based on a decreasing sliding scale system that is linked to the cumulative amount of oil produced $X c_{r f, t}^{s}$, where $i$ is the index of the corresponding tier. Therefore, for possible levels $i$ (i.e. tiers) of cumulative amount of oil produced by the end of time period $\mathrm{t}$ in scenario s, the corresponding contractor's profit oil share can be calculated using disjunction (28) where the boolean variable $Z_{r f, i, t}$ is true if the cumulative oil produced lies between the tier $\mathrm{i}$ threshold. This disjunction (28) can further be rewritten as integer and mixed-integer linear constraints (29)-(36) using the convex-hull formulation (Raman and Grossmann, 1994).

$$
\underset{i}{\vee}\left[\begin{array}{c}
Z_{r f, i, t}^{s} \\
\operatorname{ConSh}_{r f, t}^{\text {beforetax }, s}=f_{r f, i}^{P O} \cdot P O_{r f, t}^{s} \\
L_{r f, i}^{o i l} \leq x C_{r f, t}^{s} \leq U_{r f, i}^{o i l}
\end{array}\right]
$$

ConSh $_{r f, t}^{\text {beforetax }, s}=\sum_{i} D$ ConSh $_{r f, i, t}^{\text {beforetax }, s} \quad \forall r f, t, s$

$P O_{r f, t}^{s}=\sum_{i} D P O_{r f, i, t}^{s} \quad \forall r f, t, s$

$$
x c_{r f, t}^{s}=\sum_{i} D x c_{r f, i, t}^{s} \quad \forall r f, t, s
$$

$\operatorname{DConSh}_{r f, i, t}^{\text {beforetax }, s}=f_{r f, i}^{p o} \cdot D P O_{r f, i, t}^{s} \quad \forall r f, i, t, s$

$0 \leq$ DConSh $_{r f, i, t}^{\text {beforetax,s }} \leq M \cdot Z_{r f, i, t}^{s} \quad \forall r f, i, t, s$ 


$$
\begin{array}{ll}
0 \leq D P O_{r f, i, t}^{s} \leq M \cdot Z_{r f, i, t}^{s} & \forall r f, i, t, s \\
L_{r f, i}^{\text {oil }} \cdot Z_{r f, i, t}^{s} \leq D X C_{r f, i, t}^{s} \leq U_{r f, i}^{o i l} \cdot Z_{r f, i, t}^{s} & \forall r f, i, t, s \\
\sum_{i} Z_{r f, i, t}^{s}=1 & \forall r f, t, s \\
Z_{r f, i, t}^{s} \in\{0,1\} &
\end{array}
$$

The cumulative amount of oil produced from a ring-fence $r f$ by the end of time period $\mathrm{t}$ in scenario $\mathrm{s}$ is calculated as the sum of the cumulative amount of oil produced by that time period from all the fields associated to that ring-fence, eq. (37). Constraint (38) represents the total profit oil in time period t for a ring-fence $r f$ as the difference between gross revenue and the cost oil for scenario s. The gross revenues (39) in each time period t for a ring-fence $r f$ in scenario s, are computed based on the total amount of oil produced and its selling price, where total oil flow rate in time period t for ring-fence $r f$, is calculated as the sum of the oil production rates over all the fields in that ring-fence, i.e. set $F_{r f}$, in equation (40). For simplicity, we only consider the revenue generated from the oil sales, which is much larger in general as compared to the revenue from gas.

$$
\begin{array}{ll}
x C_{r f, t}^{s}=\sum_{F_{r f}} x C_{f, t}^{f i e l d, s} & \forall r f, t, s \\
P O_{r f, t}^{s}=R E V_{r f, t}^{s}-C O_{r f, t}^{s} & \forall r f, t, s \\
R E V_{r f, t}^{s}=\delta_{t} \alpha_{t} x_{r f, t}^{\text {tot }, s} & \forall r f, t, s \\
x_{r f, t}^{\text {tot,s }}=\sum_{F_{r f}} x_{f, t}^{s} & \forall r f, t, s
\end{array}
$$

The cost oil in time period t for a ring-fence $r f$, constraint (41), is calculated as the minimum of the cost recovery in that time period and maximum allowable cost oil (cost recovery ceiling) in scenario s. Eq. (41) can further be rewritten as mixed-integer linear constraints (42)(47). Cost recovery in time period t for a ring-fence $r f$ in scenario s, constraint (48), is the sum of capital and operating costs in that period t and cost recovery carried forward from previous time period $\mathrm{t}-1$. Any unrecovered cost (that is carried forward to the next period) in time period t for a 
ring-fence $r f$, is calculated as the difference between the cost recovery and cost oil in time period $\mathrm{t}$ for a scenario s (eq. (49)).

$$
\begin{array}{ll}
C O_{r f, t}^{s}=\min \left(C R_{r f, t}^{s}, f_{r f, t}^{C R} \cdot R E V_{r f, t}^{s}\right) & \forall r f, t, s \\
C O_{r f, t}^{s} \leq C R_{r f, t}^{s}+M\left(1-b_{r f, t}^{c o, s}\right) & \forall r f, t, s \\
C O_{r f, t}^{s} \geq C R_{r f, t}^{s}-M\left(1-b_{r f, t}^{c o, s}\right) & \forall r f, t, s \\
C O_{r f, t}^{s} \leq f_{r f, t}^{C R} R E V_{r f, t}^{s}+M \cdot b_{r f, t}^{c o, s} & \forall r f, t, s \\
C O_{r f, t}^{s} \geq f_{r f, t}^{C R} R E V_{r f, t}^{s}-M \cdot b_{r f, t}^{c o, s} & \forall r f, t, s \\
C O_{r f, t}^{s} \leq C R_{r f, t}^{s} & \forall r f, t, s \\
C O_{r f, t}^{s} \leq f_{r f, t}^{C R} R E V_{r f, t}^{s} & \forall r f, t, s \\
C R_{r f, t}^{s}=C A P_{r f, t}^{s}+O P E R_{r f, t}^{s}+C R F_{r f, t-1}^{s} & \forall r f, t, s \\
C R F_{r f, t}^{s}=C R_{r f, t}^{s}-C O_{r f, t}^{s} & \forall r f, t, s
\end{array}
$$

(iv) Tightening Constraints: The logic constraints (50) and (51) that define the tier sequencing are included in the model to tighten its relaxation. These constraints can be expressed as integer linear inequalities, (52) and (53), respectively, (Raman and Grossmann, 1991). In addition, the valid inequalities (54), are also included to bound the cumulative contractor's share in the cumulative profit oil by the end of time period t, based on the sliding scale profit oil share and cost oil that has been recovered (see Gupta and Grossmann, 2012b for details).

$$
\begin{array}{lr}
Z_{r f, i, t}^{s} \Rightarrow \Lambda_{\tau=t}^{T} \neg Z_{r f, i^{\prime}, \tau}^{s} & \forall r f, i, i^{\prime}<i, t, s \\
Z_{r f, i, t}^{s} \Rightarrow \bigwedge_{\tau=1}^{t} \neg Z_{r f, i^{\prime}, \tau}^{s} & \forall r f, i, i^{\prime}>i, t, s \\
Z_{r f, i, t}^{s}+Z_{r f, i^{\prime}, \tau}^{s} \leq 1 & \forall r f, i^{\prime}, i^{\prime}<i, t, t \leq \tau \leq T, s \\
Z_{r f, i, t}^{s}+Z_{r f, i^{\prime}, \tau}^{s} \leq 1 & \forall r f, i, i^{\prime}>i, t, 1 \leq \tau \leq t, s
\end{array}
$$




$$
\begin{gathered}
\sum_{\tau \leq t}\left(\text { Contsh }_{r f, \tau}^{\text {beforetax }, s} / \alpha_{\tau}\right) \leq \sum_{i^{\prime}=1}^{i^{\prime} \leq i}\left(f_{r f, i^{\prime}}^{P O}-f_{r f, i^{\prime}-1}^{P O}\right) \cdot\left(x C_{r f, t}^{s}-L_{r f, i^{\prime}}\right)-f_{r f, i^{\text {end }}}^{P O} \cdot \sum_{\tau \leq t}\left(C O_{r f, \tau}^{s} / \alpha_{\tau}\right) \\
\forall r f, i, t, s
\end{gathered}
$$

(v) Reservoir Constraints: Constraints (55)-(58) predict the reservoir behavior for each field $f$ in each time period t for a scenario s. In particular, constraint (55) restricts the oil flow rate from each well for a particular FPSO-field connection in time period t to be less than the deliverability of that field per well in scenario s. Equation (56) represents the field deliverability per well in scenario $s$ at the beginning of time period $t+1$ for a particular FPSO-field connection as the cubic equation in terms of the fractional oil recovered by the end of time period $t$ from that field. In particular, (56a) corresponds to the oil deliverability in time period 1, while (56b) corresponds to the rest of the time periods in the planning horizon. Notice that the uncertainty in the oil deliverability profile is characterized by the uncertain parameter $\alpha_{\text {oil }}^{\text {s }}$. Constraints (57) and (58) represent the separable polynomials for the cumulative water and cumulative gas produced by the end of time period t for a specific field-FPSO connection in scenario s, where $\alpha_{w c}^{s}$ and $\alpha_{g c}^{s}$ are the respective uncertain parameters. The motivation for using polynomials for cumulative water produced and cumulative gas produced as compared to WOR and GOR, is to avoid bilinear terms in the formulation, and allow converting the resulting MINLP model into an MILP formulation as explained in Gupta and Grossmann (2012a).

$$
\begin{aligned}
& x_{f, f p s o, t}^{\text {well,s }} \leq Q_{f, f p s o, t}^{d, \text { well,s }} \quad \forall f, \text { fpso,t, } s \\
& Q_{f, f p s o, 1}^{d, w e l l, s}=\alpha_{o i l}^{s} \cdot d_{1, f, f p s o} \quad \forall f, f p s o, s \\
& Q_{f, f p s o, t+1}^{d, \text { well }, s}=\alpha_{\text {oil }}^{s} \cdot\left[a_{1, f, f p s o}\left(f c_{f, t}^{s}\right)^{3}+b_{1, f, f p s o}\left(f c_{f, t}^{s}\right)^{2}+c_{1, f, f p s o} f c_{f, t}^{s}+d_{1, f, f p s o}\right] \\
& \forall f, f p s o, t<|T|, s \\
& Q_{f, f p s o, t}^{w c, s}=\alpha_{w c}^{s} \cdot\left[a_{2, f, f p s o}\left(f c_{f, t}^{s}\right)^{4}+b_{2, f, f p s o}\left(f c_{f, t}^{s}\right)^{3}+c_{2, f, f p s o}\left(f c_{f, t}^{s}\right)^{2}+d_{2, f, f p s o} f c_{f, t}^{s}\right] \\
& \forall f, f p s o, t, s \\
& Q_{f, f p s o, t}^{g c, s}=\alpha_{g c}^{s} \cdot\left[a_{3, f, f p s o}\left(f c_{f, t}^{s}\right)^{4}+b_{3, f, f p s o}\left(f c_{f, t}^{s}\right)^{3}+c_{3, f, f p s o}\left(f c_{f, t}^{s}\right)^{2}+d_{3, f, f p s o} f c_{f, t}^{s}\right] \\
& \forall f, f p s o, t, s
\end{aligned}
$$


Notice that variables $Q_{f, f p s o, t}^{w c, s}$ and $Q_{f, f p s o, t}^{g c, s}$ will be non-zero in equations (57) and (58) if $f c_{f, t}^{s}$ is non-zero even though that particular field-FPSO connection is not present. Therefore, additional constraints (59)-(66) need to be included to equate the actual cumulative water produced ( $\left.w c_{f, f p s o, t}^{s}\right)$ and cumulative gas produced ( $\left.g c_{f, f p s o, t}^{s}\right)$ for a field-FPSO connection by the end of time period t to the corresponding dummy variables $Q_{f, f p s o, t}^{w c, s}$ and $Q_{f, f p s o, t}^{g c, s}$ only if that field-FPSO connection is present in time period t, else it is zero.

$$
\begin{array}{ll}
w c_{f, f p s o, t}^{s} \leq Q_{f, f p s o, t}^{w c, s}+M_{f, f p s o}^{w c, s}\left(1-\sum_{\tau=1}^{t} b_{f, f p s o, \tau}^{s}\right) & \forall f, f p s o, t, s \\
w c_{f, f p s o, t}^{s} \geq Q_{f, f p s o, t}^{w c, s}-M_{f, f p s o}^{w c, s}\left(1-\sum_{\tau=1}^{t} b_{f, f p s o, \tau}^{s}\right) & \forall f, f p s o, t, s \\
w c_{f, f p s o, t}^{s} \leq M_{f, f p s o}^{w c, s} \sum_{\tau=1}^{t} b_{f, f p s o, \tau}^{s} & \forall f, f p s o, t, s \\
w C_{f, f p s o, t}^{s} \geq-M_{f, f p s o}^{w c, s} \sum_{\tau=1}^{t} b_{f, f p s o, \tau}^{s} & \forall f, f p s o, t, s \\
g c_{f, f p s o, t}^{s} \leq Q_{f, f p s o, t}^{g c, s}+M_{f, f p s o}^{g c, s}\left(1-\sum_{\tau=1}^{t} b_{f, f p s o, \tau}^{s}\right) & \forall f, f p s o, t, s \\
g c_{f, f p s o, t}^{s} \geq Q_{f, f p s o, t}^{g c, s}-M_{f, f p s o}^{g c, s}\left(1-\sum_{\tau=1}^{t} b_{f, f p s o, \tau}^{s}\right) & \forall f, f p s o, t, s \\
g c_{f, f p s o, t}^{s} \leq M_{f, f p s o}^{g c, s} \sum_{\tau=1}^{t} b_{f, f p s o, \tau}^{s} & \\
g c_{f, f p s o, t}^{s} \geq-M_{f, f p s o}^{g c, s} \sum_{\tau=1}^{t} b_{f, f p s o, \tau}^{s} & \forall f, f p s o, t, s
\end{array}
$$

Eq. (67) and (68) compute the water and gas flow rates in time period t from a field to FPSO facility in scenario $s$ as the difference of cumulative amounts produced by the end of current time period $\mathrm{t}$ and previous time period $\mathrm{t}-1$, divided by the time duration of that period.

$$
\begin{array}{ll}
w_{f, f p s o, t}^{s}=\left(w c_{f, f p s o, t}^{s}-w c_{f, f p s o, t-1}^{s}\right) / \delta_{t} & \forall f, f p s o, t, s \\
g_{f, f p s o, t}^{s}=\left(g c_{f, f p s o, t}^{s}-g c_{f, f p s o, t-1}^{s}\right) / \delta_{t} & \forall f, f p s o, t, s
\end{array}
$$


(vi) Field-FPSO flow constraints: The total oil flow rate in (69) from each field $f$ in time period $t$ for a scenario $s$ is the sum of the oil flow rates that are directed to FPSO facilities in that time period $\mathrm{t}$, whereas oil that is directed to a particular FPSO facility from a field $f$ in scenario $\mathrm{s}$ is calculated as the multiplication of the oil flow rate per well and number of wells available for production in that field (eq. (70)). Eq. (71) computes the cumulative amount of oil produced from field $f$ by the end of time period $t$ in scenario s, while (72) represents the fractional oil recovery by the end of time period $t$. The cumulative oil produced in scenario $\mathrm{s}$ is also restricted in (73) by the recoverable amount of oil from the field. Eqs. (74)-(76) compute the total oil, water and gas flow rates into each FPSO facility, respectively, in time period t from all the given fields in each scenario s. The total oil, water and gas flowrates in each time period $t$ for scenario $\mathrm{s}$ are calculated as the sum of the production rate of these components over all the FPSO facilities in equations (77)-(79), respectively.

$$
\begin{array}{ll}
x_{f, t}^{s}=\sum_{f p s o} x_{f, f p s o, t}^{s} & \forall f, t, s \\
x_{f, f p s o, t}^{s}=N_{f, t}^{\text {well,s }} \cdot x_{f, f p s o, t}^{\text {well,s }} & \forall f, f p s o, t, s \\
x C_{f, t}^{s}=\sum_{\tau=1}^{t}\left(x_{f, \tau}^{s} \delta_{\tau}\right) & \forall f, t, s \\
f_{f, t}^{s}=\frac{x C_{f, t}^{s}}{R E C_{f}^{s}} & \forall f, t, s \\
x C_{f, t}^{s} \leq R E C_{f}^{s} & \forall f, t, s \\
x_{f p s o, t}^{s}=\sum_{f} x_{f, f p s o, t}^{s} & \forall f p s o, t, s \\
w_{f p s o, t}^{s}=\sum_{f} w_{f, f p s o, t}^{s} &
\end{array}
$$




$$
\begin{array}{ll}
g_{f p s o, t}^{s}=\sum_{f} g_{f, f p s o, t}^{s} & \forall f p s o, t, s \\
x_{t}^{t o t, s}=\sum_{f p s o} x_{f p s o, t}^{s} & \forall t, s \\
w_{t}^{t o t, s}=\sum_{f p s o} w_{f p s o, t}^{s} & \forall t, s \\
g_{t}^{\text {tot,s }}=\sum_{f p s o} g_{f p s o, t}^{s} & \forall t, s
\end{array}
$$

(vii) FPSO Capacity Constraints: Eqs. (80)-(82) restrict the total oil, liquid and gas flow rates into each FPSO facility to be less than its corresponding capacity in each time period t, respectively. These three different kinds of capacities of a FPSO facility in time period $t$ are computed by equalities (83)-(85) as the sum of the corresponding capacity at the end of previous time period $t-1$, installation capacity at the beginning of time period $t-l_{1}$ and expansion capacity at the beginning of time period $t-l_{2}$, where $l_{1}$ and $l_{2}$ are the lead times for FPSO installation and expansions, respectively.

$$
\begin{array}{ll}
x_{f p s o, t}^{s} \leq Q_{f p s o, t}^{o i l, s} & \forall f p s o, t, s \\
x_{f p s o, t}^{s}+w_{f p s o, t}^{s} \leq Q_{f p s o, t}^{l i q, s} & \forall f p s o, t, s \\
g_{f p s o, t}^{s} \leq Q_{f p s o, t}^{g a s, s} & \forall f p s o, t, s \\
Q_{f p s o, t}^{o i l, s}=Q_{f p s o, t-1}^{o i l, s}+Q I_{f p s o, t-l_{1}}^{o i l, s}+Q E_{f p s o, t-l_{2}}^{o i l, s} & \forall f p s o, t, s \\
Q_{f p s o, t}^{l i q, s}=Q_{f p s o, t-1}^{l i q, s}+Q I_{f p s o, t-l_{1}}^{l i q, s}+Q E_{f p s o, t-l_{2}}^{l i q, s} & \forall f p s o, t, s \\
Q_{f p s o, t}^{g a s, s}=Q_{f p s o, t-1}^{g a s, s}+Q I_{f p s o, t-l_{1}}^{g a s, s}+Q E_{f p s o, t-l_{2}}^{g a s, s} & \forall f p s o, t, s
\end{array}
$$


(viii) Logic Constraints: Inequalities (86) and (87) restrict the installation and expansion of a FPSO facility to take place only once, respectively, while inequality (88) states that the connection between a FPSO facility and a field can be installed only once during the whole planning horizon. Inequality (89) ensures that a field can be connected to at most one FPSO facility in each time period t, while (90) states that at most one FPSO-field connection is possible for a field $f$ during the entire planning horizon under each scenario s. Constraints (91) and (92) state that the expansion in the capacity of a FPSO facility and the connection between a field and a FPSO facility, respectively, in time period t can occur only if that FPSO facility has already been installed by that time period.

$$
\begin{array}{ll}
\sum_{t \in T} b_{f p s o, t}^{s} \leq 1 & \forall f p s o, s \\
\sum_{t \in T} b_{f p s o, t}^{e x, s} \leq 1 & \forall f p s o, s \\
\sum_{t \in T} b_{f, f p s o, t}^{c, s} \leq 1 & \forall f, f p s o, s \\
\sum_{f p s o} b_{f, f p s o, t}^{c, s} \leq 1 & \forall f, t, s \\
\sum_{t \in T} \sum_{f p s o} b_{f, f p s o, t}^{c, s} \leq 1 & \forall f, s \\
b_{f p s o, t}^{e x, s} \leq \sum_{\tau=1}^{t} b_{f p s o, \tau}^{s} & \forall f p s o, t, s \\
b_{f, f p s o, t}^{c, s} \leq \sum_{\tau=1}^{t} b_{f p s o, \tau}^{s} & \forall f, f p s o, t, s
\end{array}
$$

(ix) Upper bounding constraints: Inequality (93) states that the oil flow rate per well from a field $f$ to a FPSO facility in time period $\mathrm{t}$ will be zero if that FPSO-field connection is not available in that time period in a scenario s. Constraints (94)-(99) are the upper-bounding constraints on the installation and expansion capacities for FPSO facilities in time period $t$ for each scenario s. The additional upper bounds on the oil, liquid and gas expansion capacities of FPSO facilities, (100)-(102), follow from the fact that these expansion capacities should be less than a certain fraction $(\mu)$ of the initial built capacities, respectively. 


$$
\begin{array}{ll}
x_{f, f p s o, t}^{\text {well,s }} \leq U_{f, f p s o}^{\text {well,oil }} \sum_{\tau=1}^{t} b_{f, f p s o, \tau}^{c, s} & \forall f, f p s o, t, s \\
Q I_{f p s o, t}^{\text {oil,s }} \leq U_{f p s o}^{o i l} b_{f p s o, t}^{s} & \forall f p s o, t, s \\
Q I_{f p s o, t}^{l i q, s} \leq U_{f p s o}^{l i q} b_{f p s o, t}^{s} & \forall f p s o, t, s \\
Q I_{f p s o, t}^{g a s, s} \leq U_{f p s o}^{g a s} b_{f p s o, t}^{s} & \forall f p s o, t, s \\
Q E_{f p s o, t}^{o i l, s} \leq U_{f p s o}^{o i l} b_{f p s o, t}^{e x, s} & \forall f p s o, t, s \\
Q E_{f p s o, t}^{l i q, s} \leq U_{f p s o}^{l i q} b_{f p s o, t}^{e x, s} & \forall f p s o, t, s \\
Q E_{f p s o, t}^{g a s, s} \leq U_{f p s o}^{g a s} b_{f p s o, t}^{e x, s} & \forall f p s o, t, s \\
Q E_{f p s o, t}^{o i l, s} \leq \mu Q_{f p s o, t-1}^{o i l, s} & \forall f p s o, t, s \\
Q E_{f p s o, t}^{l i q, s} \leq \mu Q_{f p s o, t-1}^{l i q, s} & \forall f p s o, t, s \\
Q E_{f p s o, t}^{g a s, s} \leq \mu Q_{f p s o, t-1}^{g a s, s} & \forall f p s o, t, s
\end{array}
$$

(x) Well drilling limitations: The number of wells available for production from a field in scenario s is calculated from (103) as the sum of the wells available at the end of previous time period and the number of wells drilled at the beginning of time period t. The maximum number of wells that can be drilled over all the fields during each time period $\mathrm{t}$ and in each field $f$ during complete planning horizon are restricted by the respective upper bounds in (104) and (105).

$$
\begin{array}{ll}
N_{f, t}^{\text {well }, s}=N_{f, t-1}^{\text {well,s }}+I_{f, t}^{\text {well,s }} & \forall f, t, s \\
\sum_{f} I_{f, t}^{\text {well }, s} \leq U I_{t}^{\text {well }} & \forall t, s \\
N_{f, t}^{\text {well,s }} \leq U N_{f}^{\text {well }} & \forall f, t, s
\end{array}
$$

(xi) Initial Non-anticipativity Constraints: In addition to the above constraints (1)-(105) that are equivalent to the constraints for the deterministic model with fiscal rules for each scenario $\mathrm{s}$ as in Gupta and Grossmann (2012b), we need the initial non-anticipativity constraints, eqs. (106)-(115), for time periods $T_{I} \subset T$ where the set $T_{I}$ may include only first or few initial time periods. These constraints ensure that we make the same decisions (FPSO installations, expansions and their oil, liquid, gas capacities; well drilling schedule and field-FPSO 
connections) in scenarios s and s' until uncertainty in the any of the parameters cannot be revealed.

$$
\begin{aligned}
& b_{f p s o, t}^{F P S O, s}=b_{f p s o, t}^{F P S O, s^{\prime}} \quad \forall f p s o, s, s^{\prime}, t \in T_{I} \\
& b_{f p s o, t}^{e x, s}=b_{f p s o, t}^{e x, s^{\prime}} \quad \forall f p s o, s, s^{\prime}, t \in T_{I} \\
& b_{f, f p s o, t}^{s}=b_{f, f p s o, t}^{s^{\prime}} \quad \forall f, f p s o, s, s^{\prime}, t \in T_{I} \\
& I_{f, t}^{\text {well }, s}=I_{f, t}^{\text {well,s' }} \quad \forall f, s, s^{\prime}, t \in T_{I} \\
& Q I_{f p s o, t}^{o i l, s}=Q I_{f p s o, t}^{o i l, s^{\prime}} \quad \forall f p s o, s, s^{\prime}, t \in T_{I} \\
& Q I_{f p s o, t}^{l i q, s}=Q I_{f p s o, t}^{l i q, s^{\prime}} \quad \forall f p s o, s, s^{\prime}, t \in T_{I} \\
& Q I_{f p s o, t}^{g a s, s}=Q I_{f p s o, t}^{g a s, s^{\prime}} \quad \forall f p s o, s, s^{\prime}, t \in T_{I} \\
& Q E_{f p s o, t}^{o i l, s}=Q E_{f p s o, t}^{o i l, s^{\prime}} \quad \forall f p s o, s, s^{\prime}, t \in T_{I} \\
& Q E_{f p s o, t}^{l i q, s}=Q E_{f p s o, t}^{l i q, s^{\prime}} \quad \forall f p s o, s, s^{\prime}, t \in T_{I} \\
& Q E_{f p s o, t}^{g a s, s}=Q E_{f p s o, t}^{g a s, s^{\prime}} \quad \forall f p s o, s, s^{\prime}, t \in T_{I}
\end{aligned}
$$

(xii) Conditional Non-anticipativity Constraints: To determine the scenario pairs (s, s') that are indistinguishable at the beginning of time period $t$, we consider the uncertainty resolution rule as explained in section 2.3. In particular, we assume that the uncertainty in all the parameters of a field is revealed if we drill at-least $\mathrm{N}_{1}$ number of wells in the field, and produce from that field for at-least $\mathrm{N}_{2}$ number of years. Therefore, eq. (116) is used relate the number of wells in the field to the binary variable $w_{f, t}^{1, s}$ such that the variable $w_{f, t}^{1, s}$ is true if and only if the number of wells drilled in the field are less than $\mathrm{N}_{1}$. Similarly, the production from the field $f$ has been made for less than $\mathrm{N}_{2}$ years, if and only if $w_{f, t}^{2, s}$ is true as represented in eqs. (117)-(118). The logic constraint (119) sets the value of the binary variable $w_{f, t}^{3, s}$ to be true if and only if either of $w_{f, t}^{1, s}$ or $w_{f, t}^{2, s}$ are true, i.e. uncertainty in the field $f$ has not been revealed in scenario s at the beginning of time period $t$.

$$
w_{f, t}^{1, s} \Leftrightarrow\left(N_{f, t-1}^{\text {well,s }} \leq N_{1}-1\right) \quad \forall f, t, s
$$




$$
\begin{array}{ll}
w_{f, t}^{2, s} \Leftrightarrow\left(\sum_{\tau=1}^{t-1} b_{f, \tau}^{\text {prod }, s} \leq N_{2}-1\right) & \forall f, t, s \\
b_{f, t}^{\text {prod }, s} \Leftrightarrow\left(x_{f, t}^{s} \geq \varepsilon\right) & \forall f, t, s \\
w_{f, t}^{3, s} \Leftrightarrow w_{f, t}^{1, s} \vee w_{f, t}^{2, s} & \forall f, t, s
\end{array}
$$

Based on the above value of the variable $w_{f, t}^{3, s}$, equation (120) determines the value of the boolean variable $Z_{t}^{s, s^{\prime}}$. In particular, two scenarios (s, s') will be indistinguishable at the beginning of time period $t$ if and only if for each field $f$ that distinguishes those scenarios (i.e. $\left.f \in D\left(s, s^{\prime}\right)\right), \quad w_{f, t}^{3, s}$ is true. Therefore, eqs. (116)-(120) can be used to determine the indistinguishable scenarios at the beginning of time period t based on the decisions that have been implemented before that time period. Notice that as a special case, where either well drilling or production from the field is sufficient to observe the uncertainty, then one only needs to consider eq. (116) or eqs. (117)-(118), respectively, and eq. (120) without introducing the additional variable $w_{f, t}^{3, s}$.

$$
Z_{t}^{s, s^{\prime}} \Leftrightarrow \underset{f \in D\left(s, s^{\prime}\right)}{\wedge} w_{f, t}^{3, s} \quad \forall s, s^{\prime}, t
$$

The conditional non-anticipativity constraints in disjunction (121) equate the decisions in scenarios $\mathrm{s}$ and s' for the later time periods $T_{C} \subset T$, if these scenarios are indistinguishable at the beginning of time period t, i.e. for which $Z_{t}^{s, s^{\prime}}$ is true calculated in eq. (120). 


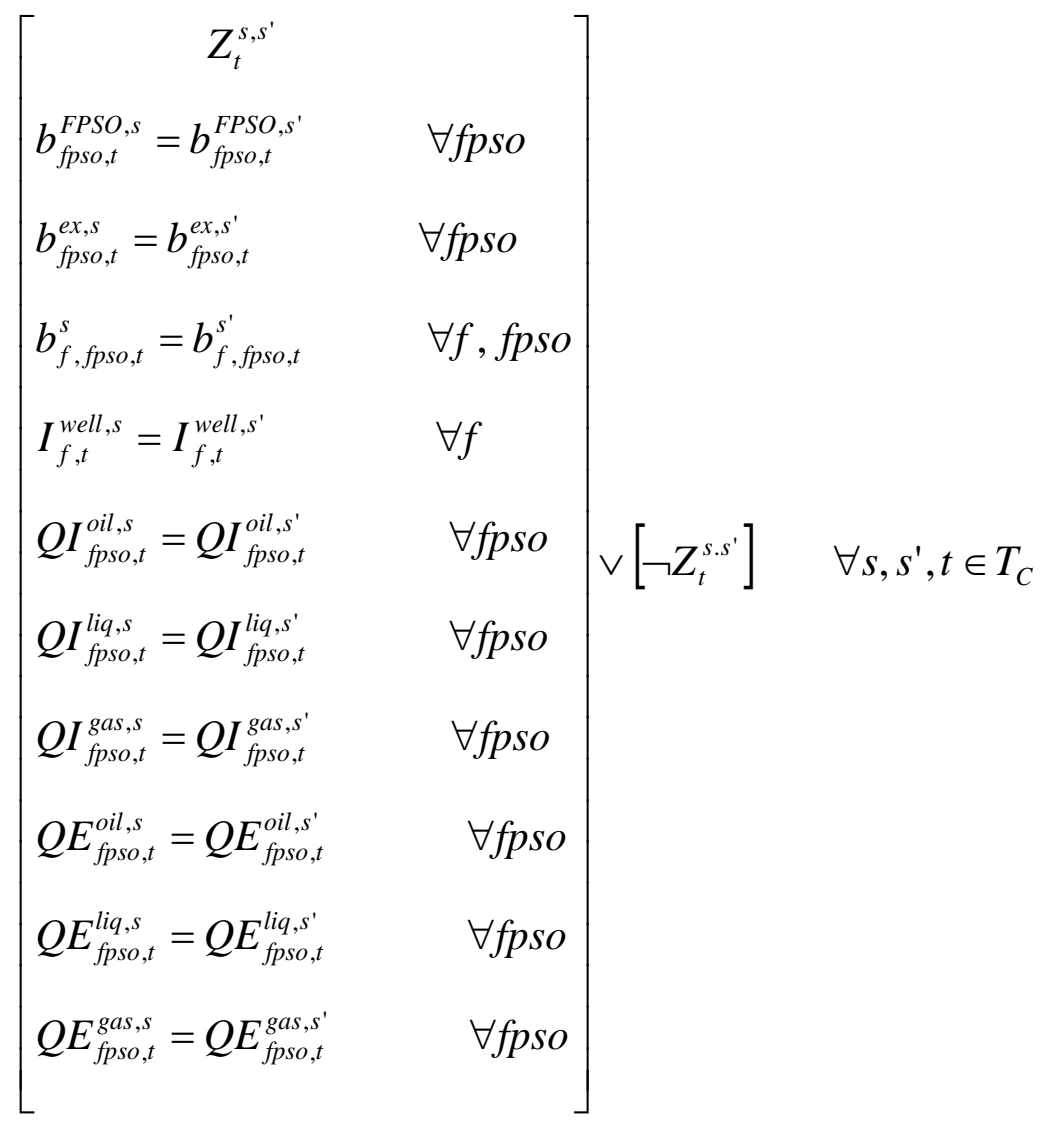

The multistage stochastic mixed-integer nonlinear disjunctive programming model (MSSPND) for offshore oilfield investment and operations planning involves constraints (1)-(13), (15)(27), (29)-(40), (42)-(49), (52)-(121) that consider endogenous uncertainty in the field parameters and sliding scale production sharing agreements with ringfencing provisions. In particular, constraints (56b)- (58) and (70) are nonlinear and non-convex constraints in the model. These constraints can be linearized using exact linearization and piecewise linear approximation techniques described in Gupta and Grossmann (2012a) to convert the nonlinear model (MSSP-ND) to a linear one (MSSP-LD). Notice that the resulting model will be an extension of the deterministic MILP fiscal model (Model 3F) in Gupta and Grossmann (2012b) to the stochastic case using the modeling framework presented in Gupta and Grossmann (2011).

\section{Compact representation of the Multistage Stochastic Model}

The proposed multistage stochastic mixed-integer linear disjunctive programming model (MSSP-LD) in the previous section can be represented in the following compact form, where all the variables in the detailed model, integer and continuous, are aggregated into the variables $X_{t}^{s}$ : 


$$
\begin{aligned}
& \text { (MD) } \quad \max \quad z=\sum_{s \in S} p^{s} \sum_{t \in T} c_{t} x_{t}^{s} \\
& \text { s.t. } \sum_{\tau \leq t} A_{\tau}^{s} x_{\tau}^{s} \leq a_{t}^{s} \quad \forall t, s \\
& x_{t}^{s}=x_{t}^{s^{\prime}} \quad \forall t \in T_{I}, \forall s, s^{\prime} \in S \\
& Z_{t}^{s, s^{\prime}} \Leftrightarrow F\left(x_{1}^{s}, x_{2}^{s} \ldots . x_{t-1}^{s}\right) \quad \forall t \in T_{C}, \forall s, s^{\prime} \in S \\
& {\left[\begin{array}{c}
Z_{t}^{s, s^{\prime}} \\
x_{t}^{s}=x_{t}^{s^{\prime}}
\end{array}\right] \vee\left[\neg Z_{t}^{s, s^{\prime}}\right] \quad \forall t \in T_{C}, \forall s, s^{\prime} \in S} \\
& x_{j t}^{s} \in I \quad \forall t, s, \forall j \in J^{\prime} \\
& x_{j t}^{s} \in R \quad \forall t, s, \forall j \in J \backslash J^{\prime}
\end{aligned}
$$

The objective function (122) in the above model (MD) maximizes the expectation of an economic criterion over the set of scenarios $s \in S$, and over a set of time periods $t \in T$, which is equivalent to eq. (1). For a particular scenario s, inequality (123) represents constraints that govern decisions $x_{t}^{s}$ in time period $t$ and link decisions across time periods. These individual scenario constraints correspond to the eqs. (2)-(13), (15)-(27), (29)-(40), (42)-(49) and (52)(105), where the nonlinear and non-convex constraints (56b)- (58) and (70) have been linearized using exact linearization and piecewise linear approximation techniques described in Gupta and Grossmann (2012a).

Non-anticipativity (NA) constraints for initial time periods $T_{I} \subset T$ are given by equations (124) for each scenario pair (s,s') to ensure the same decisions in all the scenarios, which are the compact representation for constraints (106)-(115). The conditional NA constraints are written for the later time periods $T_{C} \subset T$ in terms of logic propositions (125) and disjunctions (126). Notice that the set of initial time periods $T_{I}$ may include first few years of the planning horizon until uncertainty cannot be revealed, while $T_{C}$ represents the rest of the time periods in the planning horizon. The function $F\left(x_{1}^{s}, x_{2}^{s} \ldots x_{t-1}^{s}\right)$ in eq. (125) is an uncertainty resolution rule for a given pair of scenarios s and s' that determines the value of the corresponding boolean variable $Z_{t}^{s, s^{\prime}}$ based on the decisions that have been implemented so far as shown in eqs. (116)-(120). The 
variable $Z_{t}^{s, s^{\prime}}$ is further used in disjunction (126) to ensure the same decisions in scenarios s and $s$ ' if these are still indistinguishable in time period t, which is similar to the disjunctions (121). Equations (127)-(128) define the domain of the discrete and continuous variables in the model.

Notice that the model with a reduced number of scenario pairs (s,s') that are sufficient to represent the non-anticipativity constraints can be obtained from model (MD) after applying the three properties presented in Gupta and Grossmann (2011). These properties are defined on the basis of symmetry, adjacency and transitivity relationship among the scenarios. The reduced model (MDR) can be formulated from (MD) as follows, where $P_{3}$ is the set of minimum number of scenario pairs that are required to represent non-anticipativity in each time period t,

(MDR)

$$
\begin{gathered}
\max \quad Z=\sum_{s \in S} p^{s} \sum_{t \in T} c_{t} x_{t}^{s} \\
\text { s.t. } \quad \sum_{\tau \leq t} A_{\tau}^{s} x_{\tau}^{s} \leq a_{t}^{s} \quad \forall t, s \\
x_{t}^{s}=x_{t}^{s^{\prime}} \quad \forall t \in T_{I}, \forall\left(s, s^{\prime}\right) \in P_{3} \\
Z_{t}^{s, s^{\prime}} \Leftrightarrow F\left(x_{1}^{s}, x_{2}^{s} \ldots . x_{t-1}^{s}\right) \quad \forall t \in T_{C}, \forall\left(s, s^{\prime}\right) \in P_{3} \\
{\left[\begin{array}{r}
Z_{t}^{s, s^{\prime}} \\
x_{t}^{s}=x_{t}^{s^{\prime}}
\end{array}\right] \vee\left[\neg Z_{t}^{s, s^{\prime}}\right] \quad \forall t \in T_{C}, \forall\left(s, s^{\prime}\right) \in P_{3}} \\
x_{j t}^{s} \in I \quad \forall t, s, \forall j \in J^{\prime} \\
x_{j t}^{s} \in R \quad \forall t, s, \forall j \in J \backslash J^{\prime}
\end{gathered}
$$

The mixed-integer linear disjunctive model (MDR) can be further converted to a mixedinteger linear programming model (MLR). First, the logic constraints (130) are re-written as the mixed-integer linear constraints eq. (132) based on the uncertainty resolution rule where $Z_{t}^{s, s^{\prime}}$ is a binary variable that takes a value of 1 if scenario pair $\left(\mathrm{s}, \mathrm{s}^{\prime}\right)$ is indistinguishable in time period t, else it is zero. The disjunction (131) can then be converted to mixed-integer linear constraints (133) and (134) using the big-M formulation. The resulting mixed-integer linear model (MLR) includes constraints (122), (123), (129), (132), (133), (134), (127) and (128).

$$
B_{t}^{s} x_{t}^{s}+C_{t}^{s} z_{t}^{s, s^{\prime}} \leq d_{t}^{s} \quad \forall t \in T_{C}, \forall\left(s, s^{\prime}\right) \in P_{3}
$$




$$
\begin{aligned}
& -M\left(1-z_{t}^{s, s^{\prime}}\right) \quad \leq x_{t}^{s}-x_{t}^{s^{\prime}} \quad \forall t \in T_{C}, \forall\left(s, s^{\prime}\right) \in P_{3} \\
& M\left(1-z_{t}^{s, s^{\prime}}\right) \geq x_{t}^{s}-x_{t}^{s^{\prime}} \quad \forall t \in T_{C}, \forall\left(s, s^{\prime}\right) \in P_{3}
\end{aligned}
$$

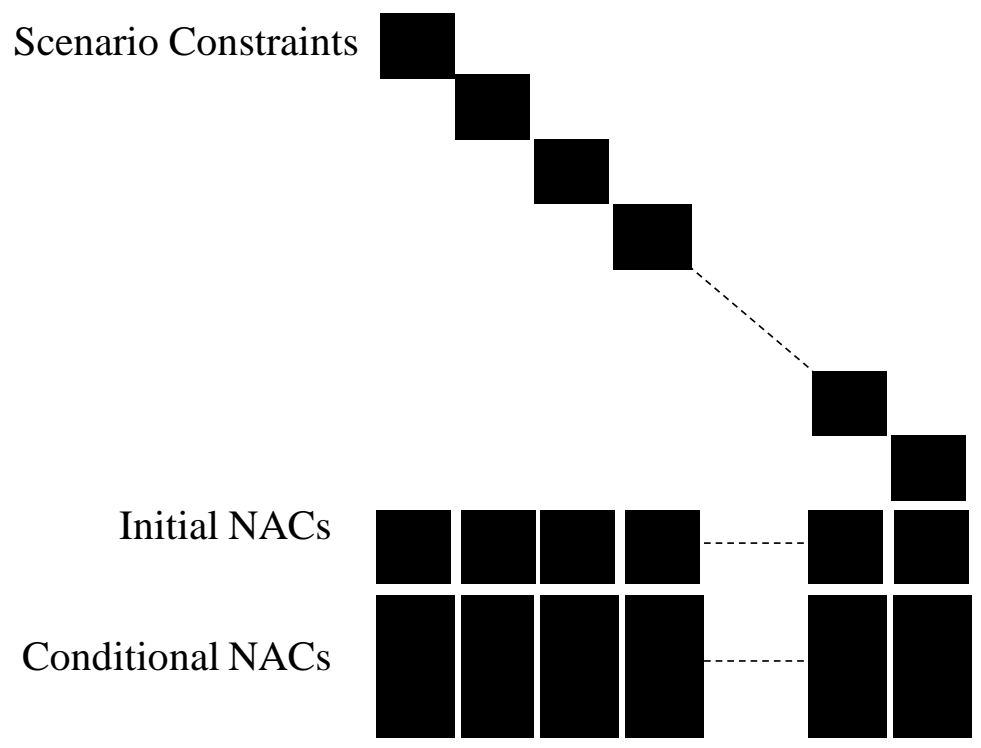

Figure 7: Structure of a typical Multistage Stochastic Program with Endogenous uncertainties

Figure 7 represents the block angular structure of model (MLR), where we can observe that the initial (eq. (129)) and conditional (eqs. (132), (133) and (134)) non-anticipativity constraints link the scenario subproblems. Therefore, these are the complicating constraints in the model. However, this structure allows decomposing the fullspace problem into smaller subproblems by relaxing the linking constraints as in Gupta and Grossmann (2011). It should be noted that the NACs (especially conditional NACs) represent a large fraction of the total constraints in the model. For clarity, we use this compact representation (MLR) in the next section to describe the solution approach instead of the detailed model (MSSP-LD) presented in the previous section.

\section{Solution Approach}

The reduced model (MLR) is composed of scenario subproblems connected through the initial (eq. (129)) and conditional (eqs. (132), (133) and (134)) non-anticipativity (NA) constraints. If these NA constraints are either relaxed or dualized using Lagrangean decomposition, then the problem decomposes into smaller subproblems that can be solved independently for each scenario within an iterative scheme for the multipliers as described in Carøe and Schultz (1999) and in Gupta and Grossmann (2011). In this way, we can effectively decompose and solve the 
large-scale oilfield development planning instances. The Lagrangean decomposition algorithm of Figure 8 for MSSP with endogenous uncertainties as proposed in Gupta and Grossmann (2011) involves obtaining the upper bound (UB) by solving the Lagrangean problem (L1-MLR) with fixed multipliers $\lambda_{t}^{s, s^{\prime}}$. The Lagrangean problem (L1-MLR) is formulated from the mixed-integer linear reduced model (MLR) by relaxing all the conditional NA constraints (132), (133) and (134), and dualizing all the initial NA constraints (129) as penalty terms in the objective function. This gives rise to the subproblems for each scenario $s \in S$, (L1-MLR $\left.{ }^{s}\right)$ that can be solved independently in parallel.

(L1-MLR)

$$
\begin{array}{cl}
\max & \sum_{s \in S} p^{s} \sum_{t \in T} c_{t} x_{t}^{s}+\sum_{t \in T_{1}\left(s, s^{\prime}\right) \in P_{3}} \lambda_{t}^{s, s^{\prime}}\left(x_{t}^{s}-x_{t}^{s^{\prime}}\right) \\
\text { s.t. } \quad & \sum_{\tau \leq t} A_{\tau}^{s} x_{\tau}^{s} \leq a_{t}^{s} \quad \forall t, s \\
& x_{j t}^{s} \in\{0,1\} \quad \forall t, s, \forall j \in J^{\prime} \\
x_{j t}^{s} \in R \quad \forall t, s, \forall j \in J \backslash J^{\prime}
\end{array}
$$

$\left(\mathbf{L} 1-\mathbf{M L R}^{s}\right) \quad \max \sum_{t \in T} p^{s} c_{t} x_{t}^{s}+\sum_{t \in T_{1}} x_{t}^{s}\left(\sum_{\substack{\left(s, s^{\prime}\right) \in P_{3} \\ s<s^{\prime}}} \lambda_{t}^{s, s^{\prime}}-\sum_{\substack{\left(s^{\prime}, s\right) \in P_{3} \\ s>s^{\prime}}} \lambda_{t}^{s^{\prime}, s}\right)$

$$
\begin{aligned}
& \text { s.t. } \sum_{\tau \leq t} A_{\tau}^{s} x_{\tau}^{s} \leq a_{t}^{s} \quad \forall t \\
& x_{j t}^{s} \in\{0,1\} \quad \forall t, \forall j \in J^{\prime} \\
& x_{j t}^{s} \in R \quad \forall t, \forall j \in J \backslash J^{\prime}
\end{aligned}
$$

The lower bound (LB) or feasible solution is generated by using a heuristic based on the solution of the Lagrangean problem (L1-MLR). In this heuristic, we fix the decisions obtained from the above problem (L1-MLR) in the reduced problem (MLR) such that there is no violation of NA constraints and solve it to obtain the lower bound. The sub-gradient method by Fisher (1985) is used during each iteration to update the Lagrangean multipliers. The algorithm, which is shown in Figure 5, stops when either a maximum iteration/time limit is reached, or the difference between the lower and upper bounds, LB and UB, is less than a pre-specified tolerance. 


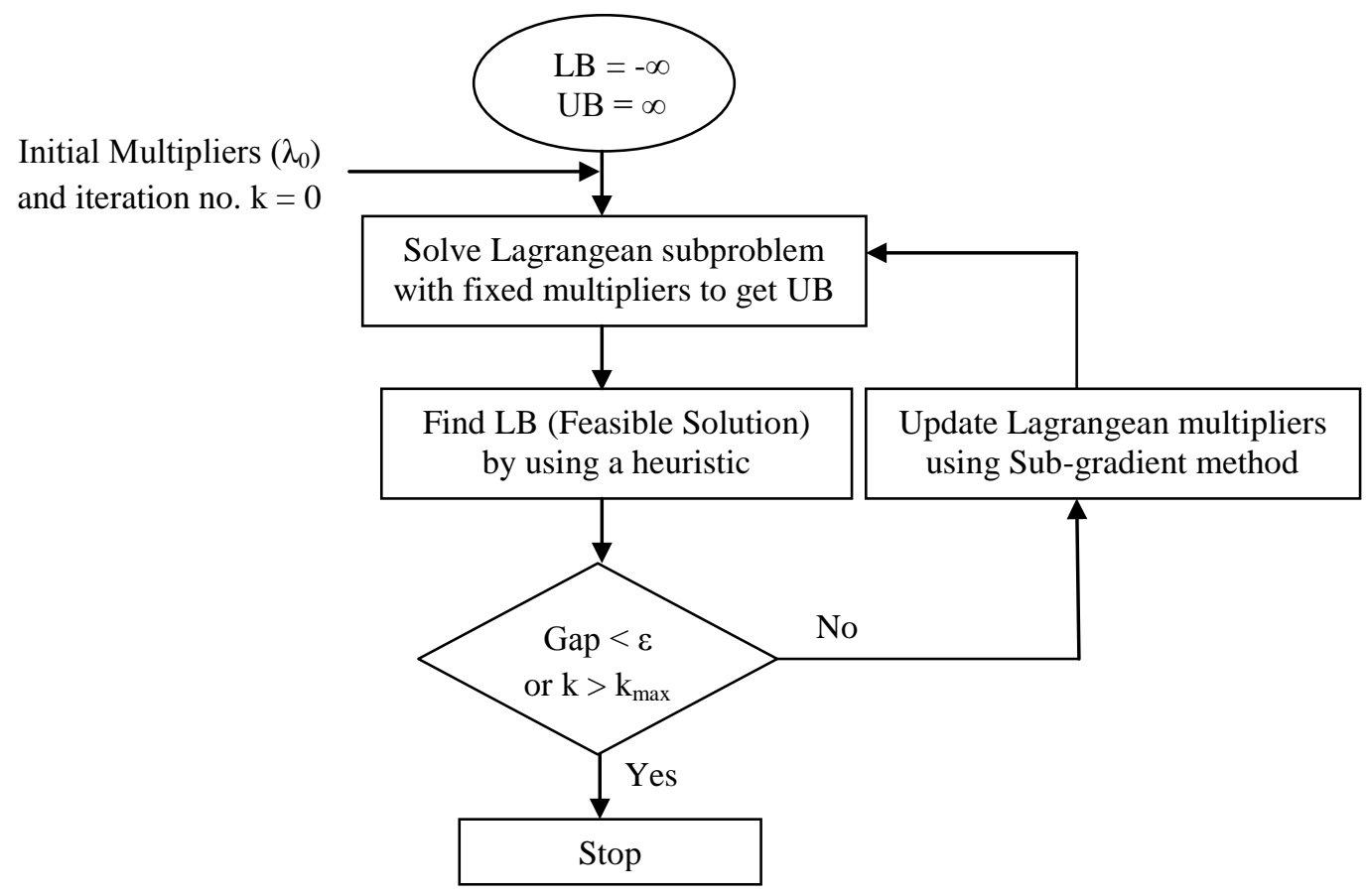

Figure 5: Lagrangean Decomposition algorithm

Notice that the extended form of this method relying on duality based branch and bound search, has also been proposed in Goel and Grossmann (2006), Tarhan et al. (2009), and Tarhan et al. (2011) to close the gap between the upper and the lower bounds. Moreover, a new Lagrangean decomposition algorithm is proposed in the next Gupta and Grossmann (2013) to further improve the quality of the dual bound at the root node.

\section{Numerical Results}

In this section, we present computational results for the offshore oilfield development planning examples under endogenous uncertainty in the field parameters, which resolves as a function of investment and operating decisions as described before. Moreover, we consider a case where progressive production sharing agreements are also present. The multistage stochastic MILP model (MLR) presented in section 4 is considered that maximizes the expected NPV value over the given planning horizon. The model is implemented in GAMS 23.6.3 and run on an Intel Core i7, 4GB RAM machine using CPLEX 12.2 solver for all the instances.

\subsubsection{Oilfield Planning Example}

\section{Case (i): Uncertainty in the field size only (4 scenarios)}


In this instance, we consider 3 oilfields and 3 potential FPSO's that can be installed. There are a total of 9 possible connections among field-FPSO (Figure 9), and 30 wells can be drilled in the fields over the planning horizon of 10 years. Field 3 has a recoverable oil volume (field size) of 500 MMbbls. However, there is uncertainty in the size of fields 1 and 2, where each one has two possible realizations (low, high) with equal probability. Therefore, there are a total of 4 scenarios each with a probability of 0.25 (see Table 1). Notice that for simplicity we only consider the cases with same probabilities for all the scenarios throughout this paper. In our future paper, if it would be possible, we will include more realistic probability values for the examples.

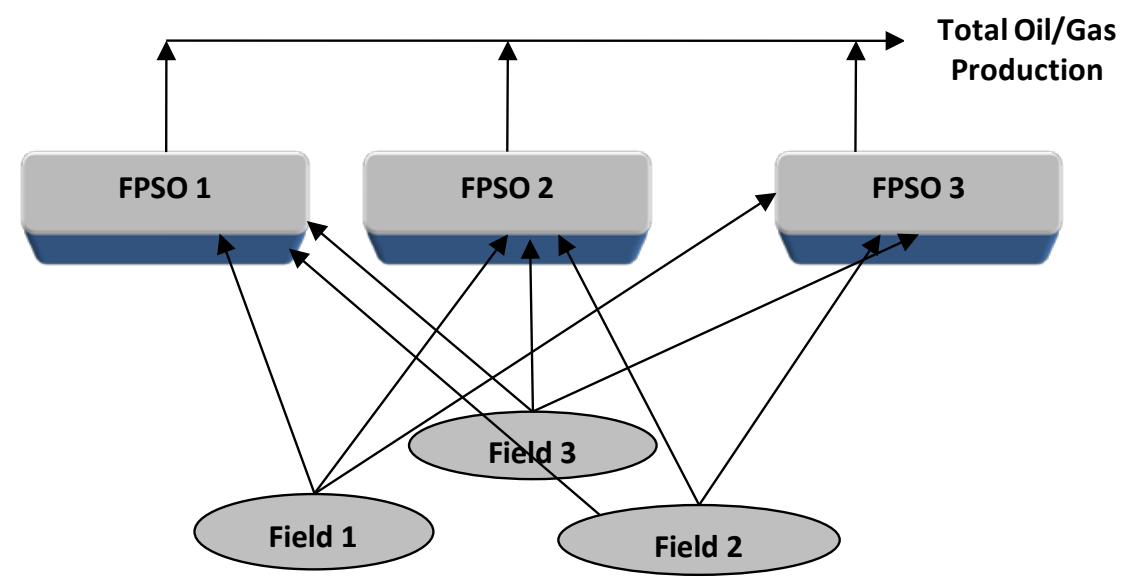

Figure 9: 3 oilfield planning example

Table 1: 3 oilfield planning example, case (i)

\begin{tabular}{|c|c|c|c|c|}
\hline Scenarios & s1 & s2 & s3 & s4 \\
\hline Field 1 Size (MMbbls) & 57 & 403 & 57 & 403 \\
\hline Field 2 Size (MMbbls) & 80 & 80 & 560 & 560 \\
\hline Scenario Probability & 0.25 & 0.25 & 0.25 & 0.25 \\
\hline
\end{tabular}

It is assumed that the uncertainty in field 1 size is revealed after drilling 3 wells $\left(\mathrm{N}_{1}=3\right)$ in the field and producing for 1 year $\left(\mathrm{N}_{2}=1\right)$ from it. Whereas, field 2 needs at-least 4 wells to be drilled $\left(\mathrm{N}_{1}=4\right)$ and one year of production $\left(\mathrm{N}_{2}=1\right)$ for this purpose. The problem is to determine the optimum investment (FPSO installations and expansions, field-FPSO connections and well drilling) and operating decisions (oil production rate) with an objective to maximize the total expected NPV (ENPV) over the planning horizon. 
Table 2: Model statistics for the 3 oilfield example, case (i)

\begin{tabular}{|c|c|c|c|c|}
\hline Problem Type & $\begin{array}{c}\text { Number of } \\
\text { Constraints }\end{array}$ & $\begin{array}{c}\text { Continuous } \\
\text { Variables }\end{array}$ & $\begin{array}{c}\text { Discrete } \\
\text { Variables }\end{array}$ & $\begin{array}{c}\text { SOS1 } \\
\text { Variables }\end{array}$ \\
\hline Reduced Model (MLR) & 16,473 & 9,717 & 876 & 240 \\
\hline Individual Scenario & 3,580 & 2,390 & 179 & 60 \\
\hline
\end{tabular}

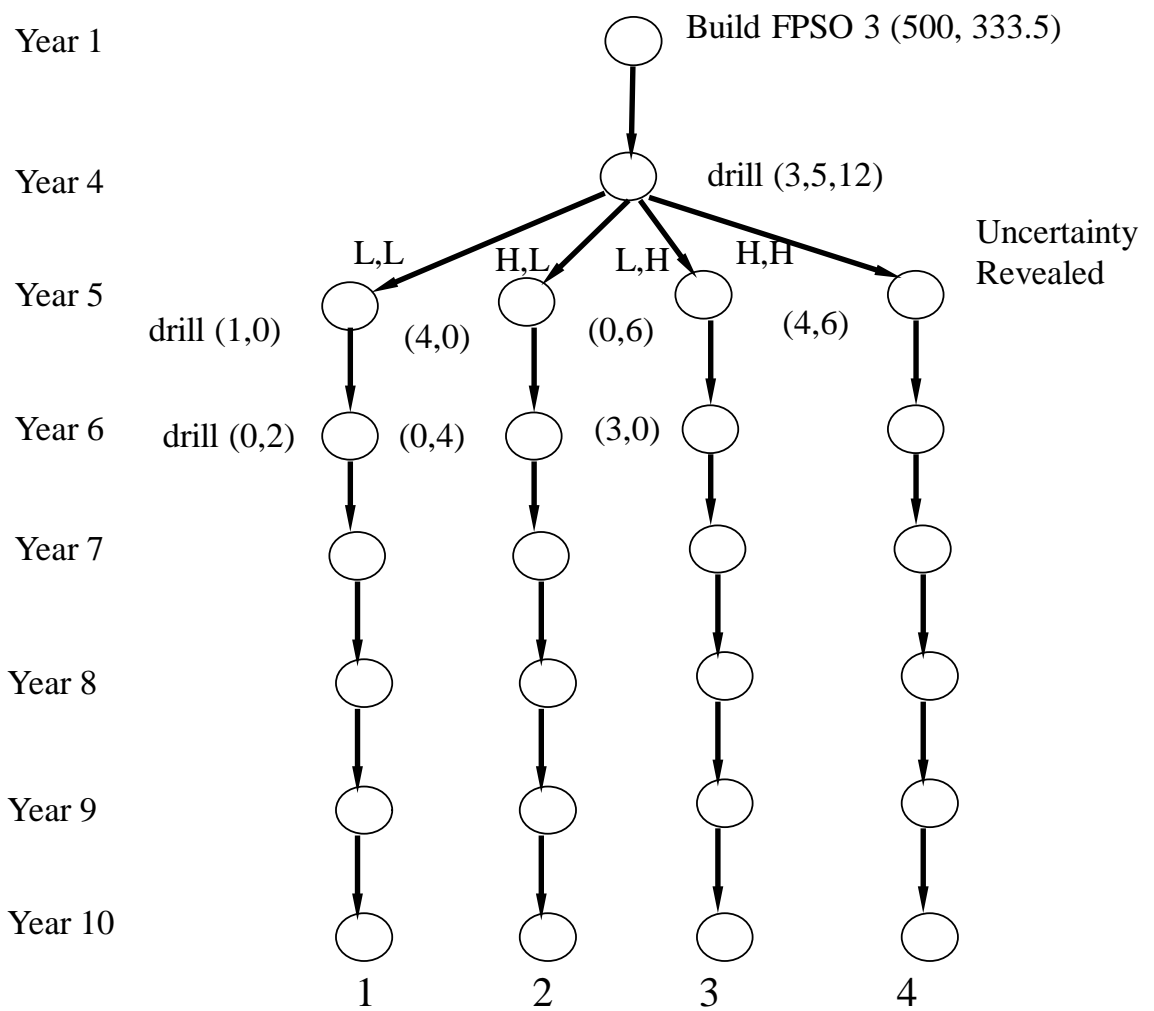

Figure 10: Optimal solution for 3 oilfield example, case (i)

The optimal ENPV for the problem is $\$ 11.50$ billion when the reduced model (MLR) is solved in fullspace using CPLEX 12.2 solver requiring 1184s. Table 2 presents the model statistics for this instance. The solution suggests installing only FPSO 3 in the first year (see Figure 10) with a capacity of $500 \mathrm{kstb} / \mathrm{d}$ and $333.5 \mathrm{MMSCF} / \mathrm{d}$ for liquid and gas, respectively. The facility is available to produce at the beginning of year 4 due to a lead time of three years. Then, we drill 3, 5 and 12 wells in fields 1,2 and 3, respectively, given the drilling-rig limitation of a total 20 wells in a year. Since, fields 1 and 2 have uncertainties, based on the realization of the uncertainty in their field sizes, more wells are drilled in these fields in the future for the favorable scenarios compared to the unfavorable outcomes, whereas no more wells are drilled in field 3. In particular, the favorable scenarios for field 1 are scenarios 2 and 4, where a total of 7 wells are drilled in the field. On the other hand, field 2 has favorable scenarios 3 and 4 , where a 
total of 11 wells are drilled in the field. Due to the different drilling and production decisions in different scenarios based on the uncertainty realizations, the capacity of FPSO3 is expanded in year 5 for scenarios 2, 3 and 4, whereas no expansion is made in the FPSO3 capacity in scenario 1. We can observe that the optimal scenario-tree is decision-dependent which is not known apriori (Figure 10).

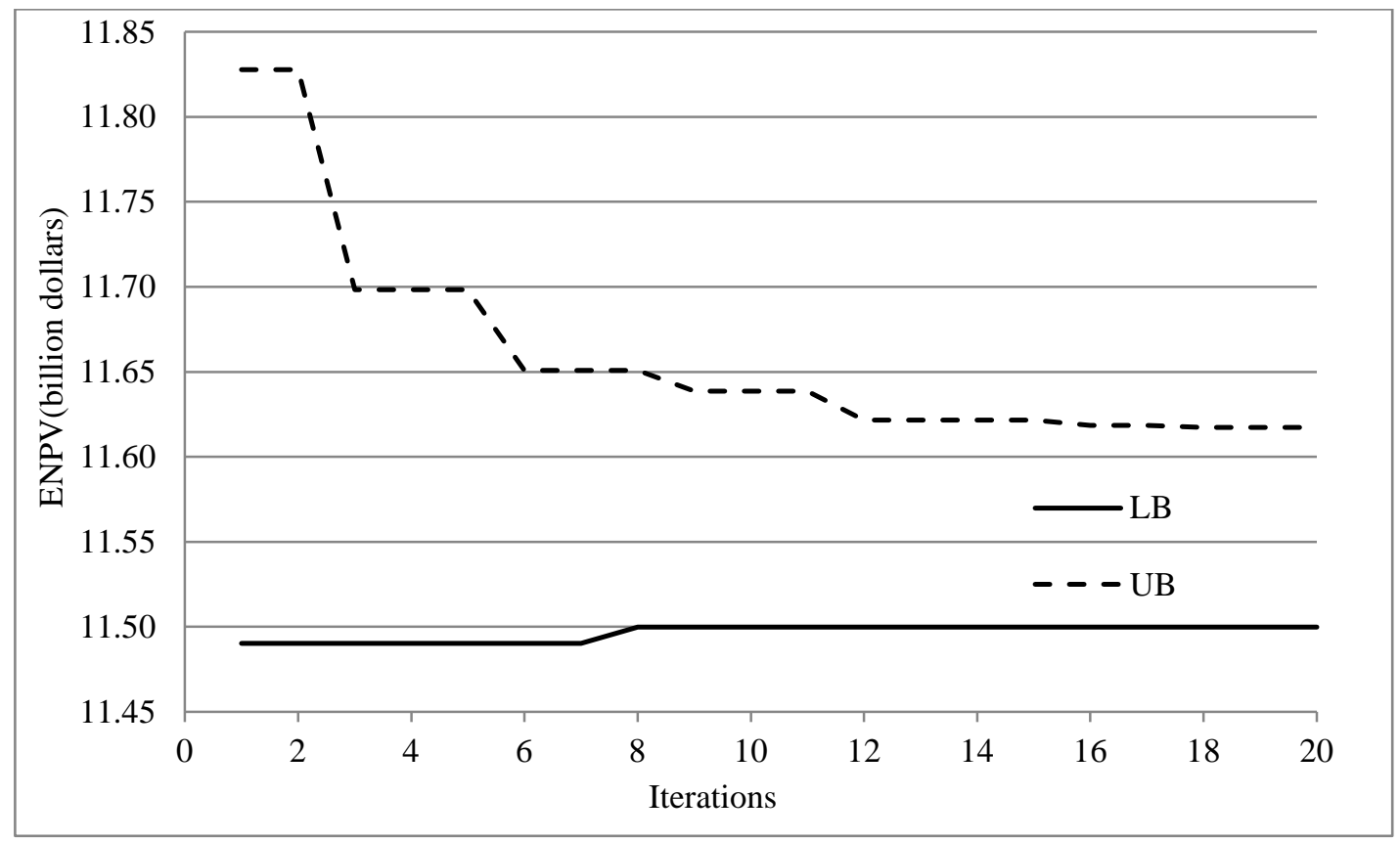

Figure 11: Lagrangean decomposition results for 3 oilfield example, case (i)

The multistage stochastic model (MLR) is also solved using the Lagrangean decomposition algorithm presented in the previous section that relies on dualizing the initial NACs and removing the conditional NACs. Figure 11 demonstrates the progress of the bounds obtained at the root node using this decomposition approach. A termination criterion of either 1\% gap or 20 sub-gradient iterations is used. We can observe that the problem can be solved in $~ 1 \%$ optimality tolerance in only 466s for the sequential implementation compared to the fullspace model that takes 1184s. Moreover, the parallel implementation of the Lagrangean decomposition algorithm in GAMS with 8 processors only takes 259s. Therefore, the proposed strategy reduces the solution time for this 4 scenario instance by more than $75 \%$ compared to the fullspace model. It is also important to note that the reformulation of the MINLP model (Model 2) to MILP approximation (Model 3) in Gupta and Grossmann (2012a) allows us to use this decomposition strategy with valid upper and lower bounds on the objective function value, without solving the 
non-convex MINLP model to global optimality which is quite expensive. Notice that the solution of the expected value problem considering the mean value of the field sizes is $\$ 11.28$ billion. Therefore, the value of the stochastic solution for this case is $\$ 220$ million or 2\%.

\section{Case (ii): Uncertainty in the field size, oil deliverability, WOR and GOR (4 scenarios)}

In this case, we consider uncertainty in the field size, oil deliverability, water-oil ratio (WOR) and gas-oil-ratio (GOR) for oilfields 1 and 2. Notice that oil deliverability, WOR and GOR are represented by the univariate polynomials in terms of the fractional oil recovery as shown in equations (143)-(145), respectively.

$$
\begin{aligned}
& Q^{d}=\alpha_{o} \cdot g\left(f_{c}\right) \\
& \text { wor }=\alpha_{w} \cdot g\left(f_{c}\right) \\
& \text { gor }=\alpha_{g} \cdot g\left(f_{c}\right)
\end{aligned}
$$

The uncertainty in oil deliverability, WOR and GOR is characterized by the uncertainty in corresponding parameters $\alpha_{o}, \alpha_{w}$ and $\alpha_{g}$. We assume that the uncertain parameters for a field are correlated, and that uncertainty in these parameters is resolved at the same time as explained earlier. This allows us to reduce a large number of scenarios in the problem. The two possible combinations of these parameters for each field results in a total of 4 scenarios each with a probability of 0.25 as can be seen in Table 3 . The data for the rest of the problem are as in case (i) presented above.

Table 4 summarizes the computational results for this case, and we can observe the similar trends as in the previous case. In particular, the fullspace multistage stochastic model using CPLEX 12.2 takes $>10,000$ s to solve the problem to optimality and it yields an expected NPV value of $\$ 11.95$ billion. The sequential and parallel implementations (8 processors) of the proposed Lagrangean decomposition approach provide a solution of $\$ 11.94$ billion with more than an order of magnitude reduction in solution times. To further reduce the gap between the upper and the lower bounds, the algorithm can be extended to the duality based branch and bound search procedure as proposed in Goel and Grossmann (2006). In addition, an improved Lagrangean decomposition approach that yields a tighter dual bound at the root node is also presented in Gupta and Grossmann (2013). 
Table 3: 3 oilfield planning example, case (ii)

\begin{tabular}{|c|c|c|c|c|c|}
\hline \multicolumn{2}{|c|}{ Scenarios } & s1 & s2 & s3 & s4 \\
\hline \multirow{4}{*}{ Field 1 } & Size (MMbbls) & 57 & 403 & 57 & 403 \\
\cline { 2 - 6 } & $\alpha_{o}$ & 0.75 & 1.25 & 0.75 & 1.25 \\
\cline { 2 - 6 } & $\alpha_{w}$ & 0.75 & 1.25 & 0.75 & 1.25 \\
\cline { 2 - 6 } & $\alpha_{g}$ & 0.75 & 1.25 & 0.75 & 1.25 \\
\hline \multirow{3}{*}{ Field 2 } & Size (MMbbls) & 80 & 80 & 560 & 560 \\
\cline { 2 - 6 } & $\alpha_{o}$ & 0.75 & 0.75 & 1.25 & 1.25 \\
\cline { 2 - 6 } & $\alpha_{w}$ & 0.75 & 0.75 & 1.25 & 1.25 \\
\cline { 2 - 6 } & $\alpha_{g}$ & 0.75 & 0.75 & 1.25 & 1.25 \\
\hline \multicolumn{2}{r|}{ Scenario Probability } & 0.25 & 0.25 & 0.25 & 0.25 \\
\hline
\end{tabular}

Table 4: Computational results for 3 oilfield example, case (ii)

\begin{tabular}{|c|c|c|c|}
\hline \multirow{2}{*}{} & \multirow{2}{*}{ Fullspace } & \multicolumn{2}{|c|}{ Lagrangean Decomposition } \\
\cline { 3 - 4 } & & Sequential & Parallel \\
\hline $\mathrm{UB}\left(\$ 10^{9}\right)$ & 11.95 & 12.14 & 12.14 \\
\hline LB $\left(\$ 10^{9}\right)$ & 11.95 & 11.94 & 11.94 \\
\hline Solution Time (s) & 10390 & 438 & 257 \\
\hline$\%$ Gap & $0 \%$ & $1.66 \%$ & $1.66 \%$ \\
\hline Subgradient iterations & - & 20 & 20 \\
\hline
\end{tabular}

\section{Case (iii): Uncertainty in the field size and progressive production sharing agreements}

We also extend the 3 oilfield example to the case where we include the progressive production sharing agreements and a planning horizon of 15 years. Table 5 represents the sliding scale profit share of the contactor involving 3 tiers that are defined on the basis of the cumulative oil production. The cost recovery ceiling of $50 \%$ of the gross revenue every year and an income tax rate of $30 \%$ is also considered. There is uncertainty in the field sizes (field 1 and 2) with a total of 4 scenarios as described in Table 1.

The multistage stochastic model becomes very difficult to solve for this instance in fullspace due to the complexities introduced in the model by the non-anticipativity constraints, and the disjunction for representing the sliding scale fiscal rules. In particular, the best solution obtained after 10 hours in fullspace using CPLEX 12.2 solver is \$2.97 billion with more than 
$21 \%$ of optimality gap (see Table 6). On the other hand, the proposed Lagrangean decomposition can solve this problem in approximately 2 hrs for the sequential implementation of the scenario subproblem solutions, and in about $1 \mathrm{hr}$ for the parallel implementation (8 processors). Both the cases yield a higher ENPV \$3.04 billion within a 0.7\% of optimality tolerance. Therefore, this example illustrates the importance of the decomposition algorithm, and its parallel implementation as more complexities are added to the problem, such as the progressive fiscal rules.

Table 5: Sliding scale contractor's profit oil share for the 3 oilfield example, case (iii)

\begin{tabular}{|c|c|c|}
\hline Tiers & Cumulative Oil Produced & Contractor's Share in Profit Oil \\
\hline Tier 1 & $0-350 \quad$ MMbbl & $50 \%$ \\
\hline Tier 2 & $350-700 \mathrm{MMbbl}$ & $40 \%$ \\
\hline Tier 3 & $>700 \quad \mathrm{MMbbl}$ & $20 \%$ \\
\hline
\end{tabular}

Table 6: Computational results for 3 oilfield example, case (iii)

\begin{tabular}{|c|c|c|c|c|c|c|c|}
\hline \multicolumn{5}{|c|}{ Fullspace Model } & \multicolumn{3}{|c|}{ Lagrangean Decomposition } \\
\hline $\begin{array}{c}\# \\
\text { Constraints }\end{array}$ & $\begin{array}{c}\text { \# } \\
\text { Dis. Var. }\end{array}$ & $\begin{array}{c}\# \\
\text { Cont. Var. }\end{array}$ & $\begin{array}{l}\text { ENPV } \\
\left(\$ 10^{9}\right)\end{array}$ & $\begin{array}{l}\text { Time } \\
\text { (s) }\end{array}$ & $\begin{array}{l}\text { ENPV } \\
\left(\$ 10^{9}\right)\end{array}$ & $\begin{array}{c}\text { Sequential } \\
\text { Time (s) }\end{array}$ & $\begin{array}{l}\text { Parallel } \\
\text { Time (s) }\end{array}$ \\
\hline 27,113 & 1,536 & 15,857 & $\begin{array}{c}\$ 2.97 \\
(>21 \%)\end{array}$ & $>36,000$ & $\begin{array}{l}\$ 3.04 \\
(0.7 \%)\end{array}$ & 8,990 & 4,002 \\
\hline
\end{tabular}

\subsection{Oilfield Planning Example}

\section{Case (i): Uncertainty in the field size only (8 scenarios)}

This is a larger example for oilfield planning problem under uncertainty than the previous one, where we consider 5 oilfields that can be connected to 3 FPSOs with 13 possible connections (Figure 12). A total of 51 wells can be drilled in the fields over the planning horizon of 20 years. There is uncertainty in the size of fields 1,3 and 5, where each one has two possible realizations (low, high) with equal probability. Therefore, there are a total of 8 scenarios each with a probability of 0.125 (see Table 7). Fields 2 and 4 have known recoverable oil volumes of 200 and 400 MMbbls, respectively.

It is assumed that the uncertainty in field 1 size is revealed after drilling 3 wells $\left(\mathrm{N}_{1}=3\right)$ in the field and producing for 1 year $\left(\mathrm{N}_{2}=1\right)$ from it. Fields 3 and 5 need at-least 4 wells to be 
drilled $\left(\mathrm{N}_{1}=4\right)$ and one year of production $\left(\mathrm{N}_{2}=1\right)$ for this purpose. The problem is to determine the optimum investment (FPSO installations and expansions, field-FPSO connections and well drilling) and operating decisions (oil production rate) with an objective to maximize the total expected NPV (ENPV) over the planning horizon.

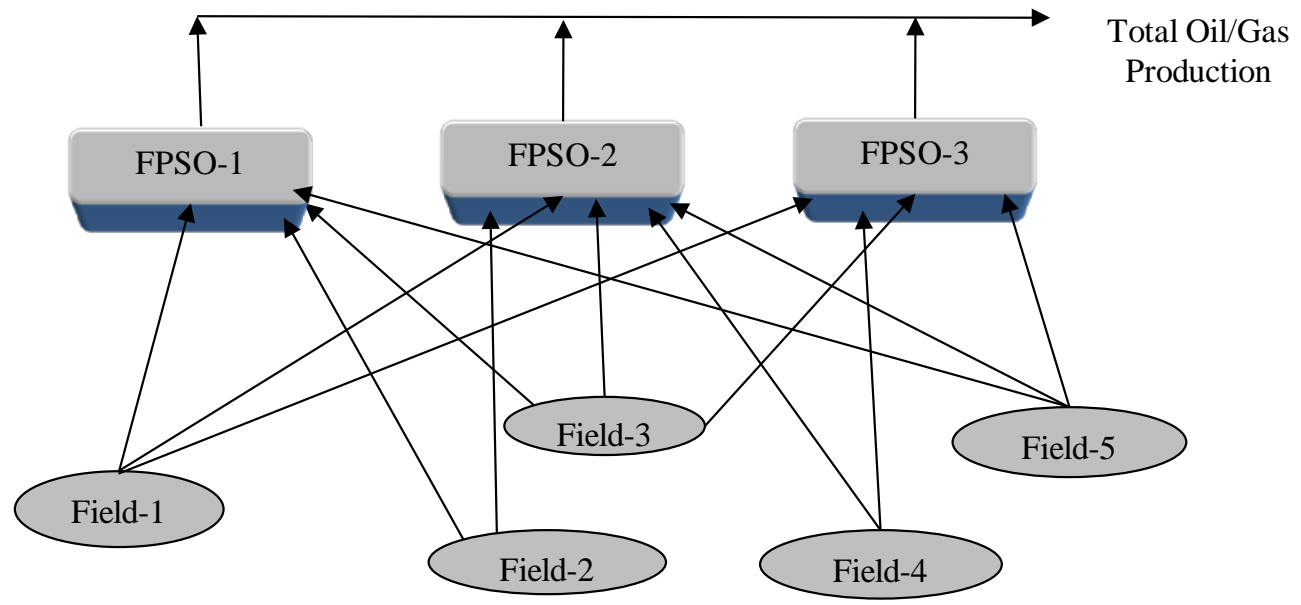

Figure 12: 5 oilfield planning example

Table 7: 5 oilfield planning example, case (i)

\begin{tabular}{|c|c|c|c|c|c|c|c|c|}
\hline Scenarios & s1 & s2 & s3 & s4 & s5 & s6 & s7 & s8 \\
\hline $\begin{array}{c}\text { Field 1 Size } \\
\text { (MMbbls) }\end{array}$ & 57 & 403 & 57 & 403 & 57 & 403 & 57 & 403 \\
\hline $\begin{array}{c}\text { Field 3 Size } \\
\text { (MMbbls) }\end{array}$ & 80 & 80 & 560 & 560 & 80 & 80 & 560 & 560 \\
\hline $\begin{array}{c}\text { Field 5 Size } \\
\text { (MMbbls) }\end{array}$ & 125 & 125 & 125 & 125 & 875 & 875 & 875 & 875 \\
\hline Scenario Probability & 0.125 & 0.125 & 0.125 & 0.125 & 0.125 & 0.125 & 0.125 & 0.125 \\
\hline
\end{tabular}

Table 8: Model statistics for the 5 oilfield example, case (i)

\begin{tabular}{|c|c|c|c|c|}
\hline Problem Type & $\begin{array}{c}\text { Number of } \\
\text { Constraints }\end{array}$ & $\begin{array}{c}\text { Continuous } \\
\text { Variables }\end{array}$ & $\begin{array}{c}\text { Discrete } \\
\text { Variables }\end{array}$ & $\begin{array}{c}\text { SOS1 } \\
\text { Variables }\end{array}$ \\
\hline Reduced Model (MLR) & 94,837 & 54,537 & 5,144 & 1600 \\
\hline Individual Scenario & 9,986 & 6,688 & 513 & 200 \\
\hline
\end{tabular}


Table 8 compares the size of the fullspace multistage stochastic MILP model with the individual scenario where a significant number of constraints and variables can be observed in the former. Therefore, the fullspace model becomes very difficult to solve directly using CPLEX 12.2 which takes more than 10 hours to reach $32 \%$ of the optimality tolerance with an expected NPV value of $\$ 20.27$ billion. The solution of the sequential implementation of the proposed Lagrangean decomposition approach also becomes expensive, but provides a solution with 3.1\% higher ENPV than the fullspace model (\$20.91 billion vs. \$20.27 billion) in 31,350s with 2.1\% of the optimality gap. The parallel implementation is the most efficient, and takes only 9,340s to yield the same objective function value as the sequential approach. Table 9 summarizes the computational results for this case, and we can observe that the impact of decomposition becomes more prominent in the larger instances. To further reduce the gap between the upper and the lower bounds, the algorithm can be extended to the duality based branch and bound search procedure as proposed in Goel and Grossmann (2006).

Table 9: Computational results for 5 oilfield example, case (i)

\begin{tabular}{|c|c|c|c|}
\hline \multirow{2}{*}{} & \multirow{2}{*}{ Fullspace } & \multicolumn{2}{|c|}{ Lagrangean Decomposition } \\
\cline { 3 - 4 } & & Sequential & Parallel \\
\hline UB $\left(\$ 10^{9}\right)$ & 26.78 & 21.37 & 21.37 \\
\hline LB $\left(\$ 10^{9}\right)$ & 20.27 & 20.91 & 20.91 \\
\hline Solution Time $(\mathrm{s})$ & $>36,000$ & 31,350 & 9,340 \\
\hline$\%$ Gap & $>32 \%$ & $2.1 \%$ & $2.1 \%$ \\
\hline Subgradient iterations & - & 20 & 20 \\
\hline
\end{tabular}

\section{Case (ii): Uncertainty in the field size, oil deliverability, WOR and GOR (8 scenarios)}

In this case, we consider uncertainty in the field size, oil deliverability, water-oil ratio (WOR) and gas-oil-ratio (GOR) for oilfields 1, 3 and 5 in Figure 12. The uncertainty in oil deliverability, water-oil ratio (WOR) and gas-oil-ratio (GOR) is characterized by the corresponding parameters, $\alpha_{o}, \alpha_{w}$ and $\alpha_{g}$ in equations (143)-(145), respectively. Two possible combinations of these parameters for each uncertain field results in a total of 8 scenarios, each with a probability of 0.125 as can be seen in Table 10. The data for the rest of the problem are similar to the case (i) presented above for 5 oilfield example. 
Table 11 represents the computational results for this case. The fullspace multistage stochastic model can only provide a solution with ENPV of $\$ 21.26$ billion in 10hrs when solved using CPLEX 12.2. The sequential as well as parallel implementation of the proposed Lagrangean decomposition approach provide a higher ENPV \$21.78 billion and a significantly tighter upper bound than the fullspace model (2.5\% gap vs. >28\% gap) in less time. Overall, the results in this case also emphasize the efficiency of the proposed Lagrangean decomposition compared to the fullspace model solved with a state-of-art commercial solver.

Table 10: 5 oilfield planning example, case (ii)

\begin{tabular}{|c|c|c|c|c|c|c|c|c|c|}
\hline \multicolumn{2}{|c|}{ Scenarios } & s1 & s2 & s3 & s4 & s5 & s6 & s7 & s8 \\
\hline \multirow{4}{*}{ Field 1 } & Size (MMbbls) & 57 & 403 & 57 & 403 & 57 & 403 & 57 & 403 \\
\cline { 2 - 10 } & $\alpha_{o}$ & 0.75 & 1.25 & 0.75 & 1.25 & 0.75 & 1.25 & 0.75 & 1.25 \\
\cline { 2 - 10 } & $\alpha_{w}$ & 0.75 & 1.25 & 0.75 & 1.25 & 0.75 & 1.25 & 0.75 & 1.25 \\
\cline { 2 - 10 } & $\alpha_{g}$ & 0.75 & 1.25 & 0.75 & 1.25 & 0.75 & 1.25 & 0.75 & 1.25 \\
\hline \multirow{5}{*}{ Field 3 } & Size (MMbbls) & 80 & 80 & 560 & 560 & 80 & 80 & 560 & 560 \\
\cline { 2 - 11 } & $\alpha_{o}$ & 0.75 & 0.75 & 1.25 & 1.25 & 0.75 & 0.75 & 1.25 & 1.25 \\
\cline { 2 - 10 } & $\alpha_{w}$ & 0.75 & 0.75 & 1.25 & 1.25 & 0.75 & 0.75 & 1.25 & 1.25 \\
\cline { 2 - 10 } & $\alpha_{g}$ & 0.75 & 0.75 & 1.25 & 1.25 & 0.75 & 0.75 & 1.25 & 1.25 \\
\cline { 2 - 10 } & Size (MMbbls) & 125 & 125 & 125 & 125 & 875 & 875 & 875 & 875 \\
\cline { 2 - 10 } & $\alpha_{o}$ & 0.75 & 0.75 & 0.75 & 0.75 & 1.25 & 1.25 & 1.25 & 1.25 \\
\cline { 2 - 9 } & $\alpha_{w}$ & 0.75 & 0.75 & 0.75 & 0.75 & 1.25 & 1.25 & 1.25 & 1.25 \\
\cline { 2 - 9 } & $\alpha_{g}$ & 0.75 & 0.75 & 0.75 & 0.75 & 1.25 & 1.25 & 1.25 & 1.25 \\
\cline { 2 - 9 } & & 0.125 & 0.125 & 0.125 & 0.125 & 0.125 & 0.125 & 0.125 & 0.125 \\
\hline
\end{tabular}

Table 11: Computational results for 5 oilfield example, case (ii)

\begin{tabular}{|c|c|c|c|}
\hline \multirow{2}{*}{} & \multirow{2}{*}{ Fullspace } & \multicolumn{2}{|c|}{ Lagrangean Decomposition } \\
\cline { 3 - 4 } & & Sequential & Parallel \\
\hline UB $\left(\$ 10^{9}\right)$ & 27.31 & 22.34 & 22.34 \\
\hline LB $\left(\$ 10^{9}\right)$ & 21.26 & 21.78 & 21.78 \\
\hline Solution Time (s) & $>36,000$ & 36,000 & 14,872 \\
\hline$\%$ Gap & $>28 \%$ & $2.5 \%$ & $2.5 \%$ \\
\hline Subgradient iterations & - & 20 & 20 \\
\hline
\end{tabular}




\section{Conclusions}

A general multistage stochastic programming model has been presented for offshore oil and gas field infrastructure planning considering endogenous uncertainties in the field parameters and progressive production sharing agreements. Discrete probability distribution functions of the uncertain parameters, i.e. field size, oil deliverability, water-oil-ratio and gas-oil ratio, are considered to represent the scenarios where uncertainty in these parameters can only be revealed once an investment is made in the field. The resulting decision-dependent scenario tree is modeled using initial and conditional non-anticipativity constraints considering the basic oilfield models developed in Gupta and Grossmann (2012a, 2012b). The model yields optimum investment and operating decisions while maximizing the expected NPV. Correlations among the endogenous uncertain parameters of a field are considered, which reduce the dimensionality of the model for large instances. The Lagrangean decomposition algorithm proposed in Gupta and Grossmann (2011) is adapted to the corresponding multistage stochastic model for oilfield development with parallel solution of the scenario subproblems. Numerical results on the two oilfield development planning examples show that the proposed Lagrangean decomposition algorithm, either sequential or parallel implementation, is computationally efficient as compared to the fullspace method, and allows the solution of intractable instances of the problem. The model and solution approach can be further used as a basis to incorporate additional complexities such as exogenous uncertainties in oil/gas prices.

Acknowledgment. The authors acknowledge financial support from ExxonMobil Upstream Research Company, Houston, TX.

\section{References}

1. Aronofsky J. S., Williams A. C., 1962. The use of linear programming and mathematical models in underground oil production. Manage. Sci., 8, 394-407.

2. Aseeri, A., Gorman, P., Bagajewicz, M. J., 2004. Financial risk management in offshore oil infrastructure planning and scheduling. Ind. Eng. Chem. Res., 43, 3063-3072.

3. Bailey, W. J., Couet, B., Wilkinson, D., 2005. Field Optimization Tool for Maximizing Asset Value. SPE Reservoir EVal. Eng., 8 (1), 7-21. 
4. Barnes, R., Linke, P., Kokossis, A., 2002. Optimization of oil-field development production capacity. European symposium on computer aided process engineering, Vol. 12 (p. 631).

5. Begg, S. H., Bratvold, R., Campbell, J. C., 2001. Improving Investment Decisions Using A Stochastic Integrated Asset Model Presented at the SPE Annual Technical Conference and Exhibition, New Orleans, LA, Sep 30-Oct 3, Paper SPE 71414.

6. Behrenbruch, P., 1993. Offshore oilfield development planning. J. Pet. Technol., 45 (8), 735-743.

7. Birge, J. R., Louveaux, F., 1997. Introduction to stochastic programming. New York, NY: Springer.

8. Blake, A. J., Roberts, M. C., 2006. Comparing petroleum fiscal regimes under oil price uncertainty. Resources Policy, 31(2), 95-105.

9. Bohannon J., 1970. A linear programming model for optimum development of multireservoir pipeline systems. J. Petrol Tech., 22, 1429-1436.

10. BP Statistical Review of World Energy, 2011.

11. Carøe, C.C., Schultz, R., 1999: Dual decomposition in stochastic integer programming. Oper. Res. Lett. 24, 37-45.

12. Carvalho, M., Pinto, J. M., 2006a. A bilevel decomposition technique for the optimal planning of offshore platforms. Brazilian J. Chem. Eng., 23, 67-82.

13. Carvalho, M., Pinto, J. M., 2006b. An MILP model and solution technique for the planning of infrastructure in offshore oilfields. J. Pet. Sci. Eng., 51, 97-110.

14. Cullick, A. S., Heath D., Narayanan K., April J., Kelly J., 2003. Optimizing Multiple-Field Scheduling and Production Strategy with Reduced Risk. Presented at the SPE Annual Technical Conference and Exhibition, Denver, CO, Oct 5-8, Paper SPE 84239.

15. Cullick, A. S., Cude, R., and Tarman, M., 2007. Optimizing Field Development Concepts for Complex Offshore Production Systems, Paper SPE 108562-MS presented at Offshore Europe, Aberdeen, Scotland, U.K., 4-7 September.

16. Dias, M., 2002. Investment in information in petroleum, real options and revelation. In: Proceedings of the 6th Annual International Conference on Real Options. Real Options Group at Cyprus, Cyprus. 
17. Ettehad, A., Jablonowski, C. J., Lake, L. W., 2011. Stochastic Optimization and Uncertainty Analysis for E\&P Projects: A Case in Offshore Gas Field Development, Offshore Technology Conference.

18. Fisher, M. L., 1985. An Applications Oriented Guide to Lagrangian Relaxation. Interfaces $15,10-21$.

19. Frair, L. C., 1973. Economic Optimization of Offshore Oilfield Development. PhD Dissertation, University of Oklahoma, Tulsa, OK.

20. Glover, F., 1975. Improved Linear Integer Programming Formulations of Nonlinear Integer Problems. Manage. Sci., 22, 455.

21. Goel, V., Grossmann, I. E., 2004. A stochastic programming approach to planning of offshore gas field developments under uncertainty in reserves. Computers and Chemical Engineering 28 (8), 1409-1429.

22. Goel, V., Grossmann, I. E., 2006. A class of stochastic programs with decision dependent uncertainty. Mathematical Programming 108 (2-3, Ser. B), 355-394.

23. Goel, V., Grossmann, I. E., El-Bakry, A. S., Mulkay, E. L., 2006. A novel branch and bound algorithm for optimal development of gas fields under uncertainty in reserves. Computers and Chemical Engineering 30, 1076-1092.

24. Gupta, V., Grossmann, I. E., 2011. Solution Strategies for Multistage Stochastic Programming with Endogenous Uncertainties. Computers and Chemical Engineering 35, 2235-2247.

25. Gupta, V., Grossmann, I. E., 2012a. An Efficient Multiperiod MINLP Model for Optimal planning of Offshore Oil and Gas Field Infrastructure. Industrial and Engineering Chemistry Research 51 (19), 6823-6840.

26. Gupta, V., Grossmann, I. E., 2012b. Modeling and Computational Strategies for Offshore Oilfield Development Planning under Complex Fiscal Rules. Industrial and Engineering Chemistry Research 51, 14438-14460.

27. Gupta, V., Grossmann, I. E., 2013. A new Decomposition Algorithm for Multistage Stochastic Programs with Endogenous Uncertainties, submitted for publication.

28. Haugen, K. K., 1996. A stochastic dynamic programming model for scheduling of offshore petroleum fields with resource uncertainty. Eur. J. Oper. Res., 88 (1), 88-100. 
29. Haugland, D., Hallefjord, A., Asheim, H., 1988. Models for petroleum field exploitation. Eur. J. Oper. Res., 37 (1), 58-72.

30. Iyer, R. R., Grossmann, I. E., Vasantharajan, S., Cullick, A. S., 1998. Optimal planning and scheduling offshore oilfield infrastructure investment and operations. Ind. Eng. Chem. Res., 37, 1380-1397.

31. Johnston, D., 1994. Petroleum Fiscal Systems and Production Sharing Contracts; PennWell Publishing Co.: Tulsa, Oklahoma.

32. Jonsbraten, T. W., 1998a. Oil-field optimization under price uncertainty. Journal of the Operational Research Society, 49, 811.

33. Jonsbraten, T. W., 1998b. Optimization models for petroleum field exploitation. PhD thesis, Norwegian School of Economics and Business Administration.

34. Jonsbraten, T. W., Wets, R. J. B., Woodruff, D. L., 1998. A class of stochastic programs with decision dependent random elements. Ann. Oper. Res., 82, 83-106.

35. Kaiser, M.J., Pulsipher, A.G., 2004. Fiscal System Analysis: Concessionary and Contractual Systems Used in Offshore Petroleum Arrangements. US Department of the Interior Mineral Management Services MMS 2004-016, 1-78.

36. Lee A. S., Aronofsky J. S., 1958. A linear programming model for scheduling crude oil production. J. Petrol Tech., 10, 51-54.

37. Lin, X., Floudas, C. A., 2003. A Novel Continuous-Time Modeling and Optimization Framework for Well Platform Planning Problems. Optim. Eng., 4 (1-2), 65-95.

38. Lund, M. W., 2000. Valuing Flexibility in Offshore Petroleum Projects. Ann. Oper. Res., 99 (1-4), 325-349.

39. Meister, B., Clark, J. M. C., Shah, N., 1996. Optimisation of oilfield exploitation under uncertainty. Computers and Chemical Engineering 20, S1251-S1256, suppl. B.

40. Ortiz-Gomez, A., Rico-Ramirez, V., Hernandez-Castro, S., 2002. Mixed-integer multiperiod model for the planning of oil-field production. Comput. Chem. Eng., 26(4-5), 703.

41. Raman, R., Grossmann, I. E., 1991. Relation between MILP Modeling and Logical Inference for Chemical Process Synthesis. Comput. Chem. Eng., 15 (2), 73-84.

42. Raman, R., Grossmann, I. E., 1994. Modeling and Computational Techniques for LogicBased Integer Programming. Comput. Chem. Eng., 18, 563-578 
43. Ruszczynski, A., 1997. Decomposition methods in stochastic programming. Math. Programming (Ser. B) 79, 333-353.

44. Schlumberger, 2008. Eclipse 2008.1 reservoir engineering software. http://www.slb.com/content/services/software/reseng/index.asp.

45. Stensland, G., Tjøstheim, D., 1991. Optimal decisions with reduction of uncertainty over time - an application to oil production. In: Lund, D., øksendal, B. (Eds.), Stochastic Models and Option Values. pp. 267-291.

46. Sullivan J., 1982. A computer model for planning the development of an offshore gas field. J. Petrol Tech., 34, 1555-1564.

47. Tarhan, B., Grossmann, I. E., Goel, V., 2009. Stochastic programming approach for the planning of offshore oil or gas field infrastructure under decision-dependent uncertainty. Industrial and Engineering Chemistry Research 48 (6), 3078-3097.

48. Tarhan, B., Grossmann, I. E., Goel, V., 2011. Computational strategies for non-convex multistage MINLP models with decision-dependent uncertainty and gradual uncertainty resolution. Annals of Operations Research, http://dx.doi.org/10.1007/s10479-011-0855-x.

49. Tsarbopoulou, C., 2000. Optimization of oil facilities and oil production. M.Sc. Dissertation, University College London, London, U.K..

50. van den Heever, S. A., Grossmann, I. E., 2000. An iterative aggregation/disaggregation approach for the solution of a mixed integer nonlinear oilfield infrastructure planning model. Ind. Eng. Chem. Res., 39, 1955-1971.

51. van den Heever, S. A., Grossmann, I. E., Vasantharajan, S., Edwards, K., 2000. Integrating complex economic objectives with the design and planning of offshore oilfield infrastructures. Comput. Chem. Eng., 24, 1049-1055.

52. van den Heever, S. A., Grossmann, I. E., 2001. A Lagrangean Decomposition Heuristic for the Design and Planning of Offshore Hydrocarbon Field Infrastructures with Complex Economic Objectives. Ind. Eng. Chem. Res., 40, 2857-2875.

53. World Bank, 2007. Contracts for Petroleum Development - Part 1-3. Petroleum Sector Briefing Note No. 7.

54. Zabalza-Mezghani, I., Manceau, E., Feraille, M., Jourdan, A., 2004. Uncertainty management: From geological scenarios to production scheme optimization. J. Pet. Sci. Eng., $44,11-25$. 